\title{
Chaotic Dynamics and Control of Discrete Ratio-Dependent Predator-Prey System
}

\author{
Sarker Md. Sohel Rana \\ Department of Mathematics, University of Dhaka, Dhaka 1000, Bangladesh \\ Correspondence should be addressed to Sarker Md. Sohel Rana; srana.mthdu@gmail.com
}

Received 30 March 2017; Revised 8 May 2017; Accepted 15 May 2017; Published 10 July 2017

Academic Editor: Abdelalim Elsadany

Copyright (C) 2017 Sarker Md. Sohel Rana. This is an open access article distributed under the Creative Commons Attribution License, which permits unrestricted use, distribution, and reproduction in any medium, provided the original work is properly cited.

This study examines the complexity of a discrete-time predator-prey system with ratio-dependent functional response. We establish algebraically the conditions for existence of fixed points and their stability. We show that under some parametric conditions the system passes through a bifurcation (flip or Neimark-Sacker). Numerical simulations are presented not only to justify theoretical results but also to exhibit new complex behaviors which include phase portraits, orbits of periods 9,19 , and 26, invariant closed circle, and attracting chaotic sets. Moreover, we measure numerically the Lyapunov exponents and fractal dimension to confirm the chaotic dynamics of the system. Finally, a state feedback control method is applied to control chaos which exists in the system.

\section{Introduction}

The interaction between predator and prey is one of the most studied topics in ecology and mathematical biology. The Lotka-Volterra model $[1,2]$ has received more attention from mathematician and ecologist. After them, many authors qualitatively analysed other types of predator-prey models in which response function of predator depends on prey densities only. But a number of respectable researchers [35] have claimed that in some environments (when predator needs to search and share foods), the response function of predator may depend on the ratio of prey to predator abundance. For detailed results in predator-prey systems with ratio-dependent response function, we refer to [6-9]. However, when the size of populations is small, the discretization of predator-prey systems is more suitable compared to continuous ones to understand unpredictable dynamic behaviors which exist in the system. Lots of empirical and theoretical works [10-20] have discussed the bifurcations and chaos phenomenon by using numerical simulations or center manifold theory and bifurcation theory.

In recent times, there are a few number of articles discussing the dynamics of ratio-dependent discrete-time predator-prey systems [21-23]. For discrete ratio-dependent predator-prey systems, it is shown that positive equilibrium is globally asymptotically stable [21], the strong and the weak Allee effects are investigated in [22], and it is discussed that periodic solutions exist in [23].

A ratio-dependent predator-prey system takes the form:

$$
\begin{aligned}
& \dot{x}=x g(x)-c f\left(\frac{x}{y}\right) y, \\
& \dot{y}=y\left(-D+e f\left(\frac{x}{y}\right)\right),
\end{aligned}
$$

where $x$ and $y$ stand for densities of prey and predator, respectively, $D>0$ is predator's death rate, and $g(x)$ is prey's specific growth rate. $f(x / y)$ is ratio-dependent response function of predator and $c, e>0$ are rate of capturing and conversion, respectively.

If $g(x)=r(1-x / K)$ and $f(x)=\alpha x /(\beta+x)$, then system (1) takes in the form [6]:

$$
\begin{aligned}
& \dot{x}=r x\left(1-\frac{x}{K}\right)-\frac{c \alpha x y}{\beta y+x}, \\
& \dot{y}=y\left(-D+\frac{e \alpha x}{\beta y+x}\right),
\end{aligned}
$$


where $r, K, \alpha$, and $\beta$ are all positive constants. By scaling the variables and parameters as $x \rightarrow x / K, y \rightarrow \beta y / K$, and $t \rightarrow$ $r t$, we write system (2) as

$$
\begin{aligned}
& \dot{x}=x(1-x)-\frac{a x y}{y+x}, \\
& \dot{y}=y\left(-d+\frac{b x}{y+x}\right)
\end{aligned}
$$

with $a=c \alpha / \beta r, b=e \alpha / r$, and $d=D / r$ being positive constants. Forward Euler scheme is applied to system (3) to obtain the following system:

$$
H:\left(\begin{array}{l}
x \\
y
\end{array}\right) \rightarrow\left(\begin{array}{c}
x+\delta\left[x(1-x)-\frac{a x y}{y+x}\right] \\
y+\delta\left[-d y+\frac{b x y}{y+x}\right]
\end{array}\right)
$$

where $\delta$ is the step size. The motivation of this work is to study systematically the dynamical properties of (4) in detail which includes phase portraits, bifurcation in orbits of periods 9 , 19, and 26, chaotic sets, Lyapunov exponents, and fractal dimension.

This paper is organized as follows. Section 2 deals with the existence condition for fixed points of (4) and their stability criterion. In Section 3, we prove that system (4) undergoes a flip bifurcation and a NS bifurcation in the interior of $\mathbb{R}_{+}^{2}$ when $\delta$ changes its value in a small neighborhood of a specific parametric space. In Section 4, we provide numerical simulations for one or more control parameters to validate analytical results. In Section 5, we use the method of feedback control to stabilize chaos at the state of unstable trajectories. Finally, a brief discussion is carried out in Section 6 .

\section{Fixed Points: Existence and Their Stability}

The fixed points of (4) are the solution of the following equations:

$$
\begin{array}{r}
x+\delta\left[x(1-x)-\frac{a x y}{y+x}\right]=x, \\
y+\delta\left[-d y+\frac{b x y}{y+x}\right]=y .
\end{array}
$$
result.

A simple algebraic computation yields the following

Lemma 1. System (4) has two fixed points: exists;

(i) the predator free fixed point $E_{1}(1,0)$, which always

(ii) the interior fixed point $E_{2}\left(x^{*}, y^{*}\right)$, where $x^{*}=1+$ $a(-1+d / b)$ and $y^{*}=((b-d) / d)[1+a(-1+d / b)]$. This fixed point exists if $d<b<a d /(a-1)$ with $a>1$.

To show the region in the space $(d, a, b)$ for which positive fixed point of system (4) exists, we plot $C=\{(d, a, b): d<$ $b<a d /(a-1)$ with $a>1\}$ in Figure 1. We see that $E_{2}$ lies inside $\mathscr{C}$ but not in regions (I)-(II).

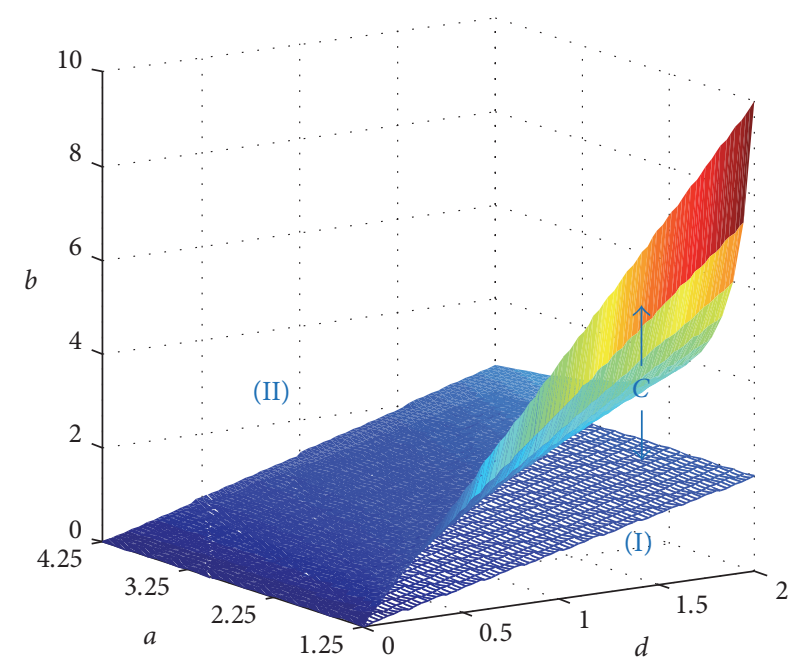

FIgURE 1: Graphical depiction for positive fixed point.

Next, we investigate stability of (4) at fixed points. The Jacobian matrix of system (4) at fixed point $E(x, y)$ is

$$
J(x, y)=\left(\begin{array}{cc}
1+\delta a_{1} & -\delta b_{1} \\
\delta a_{2} & 1+\delta b_{2}
\end{array}\right),
$$

where

$$
\begin{aligned}
& a_{1}=1-\frac{a y}{x+y}+x\left(-2+\frac{a y}{(x+y)^{2}}\right), \\
& b_{1}=\frac{a x^{2}}{(x+y)^{2}}, \\
& a_{2}=\frac{b y^{2}}{(x+y)^{2}}, \\
& b_{2}=\frac{b x^{2}}{(x+y)^{2}}-d .
\end{aligned}
$$

Then the characteristic equation of (6) is

$$
F(\lambda):=\lambda^{2}-(\operatorname{tr} J) \lambda+\operatorname{det} J=0,
$$

where

$$
\begin{aligned}
\operatorname{tr} J & =2+\delta\left(a_{1}+b_{2}\right) \\
\operatorname{det} J & =1+\delta\left(a_{1}+b_{2}\right)+\delta^{2}\left(a_{1} b_{2}+a_{2} b_{1}\right) .
\end{aligned}
$$

It is noted that the magnitude of eigenvalues of Jacobian matrix evaluated at fixed point $E(x, y)$ determines the local stability of that fixed point. The following propositions represent the stability conditions of fixed points by Jury's criterion [24].

Proposition 2. For all permissible parameters values, there are four different topological types of $E_{1}$ and it is a

(i) $\operatorname{sink}$ if $0<\delta<2$ and $d-2 / \delta<b<d$; 
(ii) source if $\delta>2$ and $(b<d-2 / \delta$ and $b>d)$;

(iii) nonhyperbolic if $\delta=2$ or $(b=d-2 / \delta$ and $b \neq d)$;

(iv) saddle if otherwise.

Condition (iii) can be written as

$$
\begin{aligned}
& \mathrm{FB}_{E_{1}}^{1}=\left\{(a, b, d, \delta) \in(0,+\infty): \delta=2, b \neq d-\frac{2}{\delta}, b\right. \\
& \quad \neq d\}
\end{aligned}
$$

or

$$
\begin{aligned}
& \mathrm{FB}_{E_{1}}^{2}=\left\{(a, b, d, \delta) \in(0,+\infty): b=d-\frac{2}{\delta}, \delta \neq 2, b\right. \\
& \quad \neq d\} .
\end{aligned}
$$

It follows that if parameters $(a, b, d, \delta)$ belong to $\mathrm{FB}_{E_{1}}^{1}$ or $\mathrm{FB}_{E_{1}}^{2}$, then two eigenvalues of $J\left(E_{1}\right)$ are $\lambda_{1}=-1$ and $\lambda_{2} \neq-1,1$. Thus a flip bifurcation may occur if parameters belong to $\mathrm{FB}_{E_{1}}^{1}$ or $\mathrm{FB}_{E_{1}}^{2}$. This observation illustrates that predator goes to extinct and prey passes through flip bifurcation leading to chaos as bifurcation parameter $\delta$ varies.

Proposition 3. If $d<b<a d /(a-1)$ with $a>1$, then positive fixed point $E_{2}$ of (4) exists and it is a

(i) sink if either of the following conditions holds:

(i.1) $P^{*} \geq 0$ and $\delta<\left(N^{*}-\sqrt{P^{*}}\right) / M^{*}$;

(i.2) $P^{*}<0$ and $\delta<N^{*} / M^{*}$;

(ii) source if either of the following conditions holds:

(ii.1) $P^{*} \geq 0$ and $\delta>\left(N^{*}+\sqrt{P^{*}}\right) / M^{*}$;

(ii.2) $P^{*}<0$ and $\delta>N^{*} / M^{*}$;

(iii) nonhyperbolic if either of the following conditions holds:

(iii.1) $P^{*} \geq 0$ and $\delta=\left(N^{*} \pm \sqrt{P^{*}}\right) / M^{*}$;

(iii.2) $P^{*}<0$ and $\delta=N^{*} / M^{*}$;

(iv) saddle if otherwise,

where

$$
\begin{aligned}
& M^{*}=a_{1} b_{2}+a_{2} b_{1}, \\
& N^{*}=-\left(a_{1}+b_{2}\right), \\
& P^{*}=N^{* 2}-4 M^{*} .
\end{aligned}
$$

From Proposition 3, it follows that if term (iii.1) holds, two eigenvalues of $J\left(E_{2}\right)$ are $\lambda_{1}=-1$ and $\lambda_{2} \neq-1,1$. We rewrite the term (iii.1) as follows:

$$
\begin{aligned}
& \mathrm{FB}_{E_{2}}^{1}=\left\{(a, b, d, \delta) \in(0,+\infty): \delta=\frac{N^{*}-\sqrt{P^{*}}}{M^{*}}, P^{*}\right. \\
& \quad \geq 0\},
\end{aligned}
$$

or

$$
\begin{aligned}
& \mathrm{FB}_{E_{2}}^{2}=\left\{(a, b, d, \delta) \in(0,+\infty): \delta=\frac{N^{*}+\sqrt{P^{*}}}{M^{*}}, P^{*}\right. \\
& \quad \geq 0\} .
\end{aligned}
$$

Therefore, a flip bifurcation may emerge from fixed point $E_{2}$ if parameters belong to $\mathrm{FB}_{E_{2}}^{1}$ or $\mathrm{FB}_{E_{2}}^{2}$.

Also two eigenvalues $\lambda_{1,2}$ of $J\left(E_{2}\right)$ are complex conjugate having magnitude one if (iii.2) holds. We rewrite the term (iii.2) as follows:

$$
\mathrm{NSB}_{E_{2}}=\left\{(a, b, d, \delta) \in(0,+\infty): \delta=\frac{N^{*}}{M^{*}}, P^{*}<0\right\},
$$

and if the parameters lie in $\mathrm{NSB}_{E_{2}}$, there exists a NS bifurcation emerging from $E_{2}$.

\section{Analysis of Bifurcation}

In this section, attention is paid to recapitulate bifurcations (flip and/or Neimark-Sacker) of system (4) around fixed points using the theories in center manifold and of bifurcation [25-28]. The parameter $\delta$ is chosen as a bifurcation parameter.

3.1. Flip Bifurcation at Fixed Point $E_{1}(1,0)$. We consider system (4) at fixed point $E_{1}$ and take parameter $(a, b, d, \delta)$ arbitrarily from $\mathrm{FB}_{E_{1}}^{1}$ (similarly for the case of $\mathrm{FB}_{E_{1}}^{2}$ ).

Let $\delta=\delta_{1}=2$, or $\delta=\delta_{1}=-2 /(b-d)$, for the case of $\mathrm{FB}_{E_{1}}^{2}$. Then the eigenvalues of positive fixed point $E_{1}(1,0)$ are

$$
\begin{aligned}
& \lambda_{1}\left(\delta_{1}\right)=-1, \\
& \lambda_{2}\left(\delta_{1}\right)=1+2(b-d) .
\end{aligned}
$$

The condition $\left|\lambda_{2}\left(\delta_{1}\right)\right| \neq 1$ holds if

$$
b-d \neq 0,-1 \text {. }
$$

Using the transformation $\tilde{x}=x-1, \tilde{y}=y$ and writing $A(\delta)=J(1,0)$, we shift the fixed point $(1,0)$ of system (4) to the origin. After Taylor expansion, system (4) reduces to

$$
\left(\begin{array}{c}
\tilde{x} \\
\tilde{y}
\end{array}\right) \longrightarrow A(\delta)\left(\begin{array}{l}
\tilde{x} \\
\tilde{y}
\end{array}\right)+\left(\begin{array}{l}
F_{1}(\tilde{x}, \tilde{y}, \delta) \\
F_{2}(\tilde{x}, \tilde{y}, \delta)
\end{array}\right),
$$

where $X=(\tilde{x}, \tilde{y})^{T}$ and

$$
\begin{aligned}
F_{1}(\tilde{x}, \tilde{y}, \delta)= & \left(-a \tilde{x} \tilde{y}^{2}-a \tilde{y}^{3}-\tilde{x}^{2}+a \tilde{y}^{2}\right) \delta \\
& +O\left(\|X\|^{4}\right), \\
F_{2}(\tilde{x}, \tilde{y}, \delta)= & \left(b \tilde{x} \tilde{y}^{2}+b \tilde{y}^{3}-b \tilde{y}^{2}\right) \delta+O\left(\|X\|^{4}\right) .
\end{aligned}
$$


Therefore, we get the following symmetric multilinear vector functions of $x, y, u \in \mathbb{R}^{2}$ :

$$
\begin{aligned}
& B(x, y)=\left(\begin{array}{l}
B_{1}(x, y) \\
B_{2}(x, y)
\end{array}\right)=\left(\begin{array}{c}
\left(2 a x_{2} y_{2}-2 x_{1} y_{1}\right) \delta \\
-2 b x_{2} y_{2} \delta
\end{array}\right), \\
& C(x, y, u)=\left(\begin{array}{l}
C_{1}(x, y, u) \\
C_{2}(x, y, u)
\end{array}\right) \\
& =\left(\begin{array}{c}
\left(-2 x_{2} y_{1} u_{2}-2 x_{1} y_{2} u_{2}-2 x_{2} y_{2} u_{1}-6 x_{2} y_{2} u_{2}\right) a \delta \\
\left(2 x_{2} y_{1} u_{2}+2 x_{1} y_{2} u_{2}+2 x_{2} y_{2} u_{1}+6 x_{2} y_{2} u_{2}\right) b \delta
\end{array}\right),
\end{aligned}
$$

where

$$
\begin{aligned}
& B_{i}(x, y)=\left.\sum_{j, k=1}^{2} \frac{\delta^{2} F_{i}(\xi, \delta)}{\delta \xi_{j} \delta \xi_{k}}\right|_{\xi=0} x_{j} y_{k}, \\
& C_{i}(x, y, u)=\left.\sum_{j, k, l=1}^{2} \frac{\delta^{2} F_{i}(\xi, \delta)}{\delta \xi_{j} \delta \xi_{k} \delta \xi_{l}}\right|_{\xi=0} x_{j} y_{k} u_{l}, \\
&(i=1,2), \delta=\delta_{1} .
\end{aligned}
$$

Let $p, q \in \mathbb{R}^{2}$ be two eigenvectors (left and right) of $A$ associated with eigenvalue $\lambda_{1}\left(\delta_{1}\right)=-1$, respectively. Then $A\left(\delta_{1}\right) q=-q$ and $A^{T}\left(\delta_{1}\right) p=-p$. After some algebraic calculation, we get

$$
\begin{aligned}
& q \sim\left(2+\delta_{1}(b-d), 0\right)^{T}, \\
& p \sim\left(2+\delta_{1}(b-d), 2 a\right)^{T} .
\end{aligned}
$$

We set $p=\gamma_{1}\left(2+\delta_{1}(b-d), 2 a\right)^{T}$, where $\gamma_{1}=1 /\left(2+\delta_{1}(b-\right.$ $d))^{2}$. Then by the standard scalar product in $\mathbb{R}^{2}$ defined by $\langle p, q\rangle=p_{1} q_{1}+p_{2} q_{2}$, it is obvious to see that $\langle p, q\rangle=1$. The direction of the flip bifurcation can be obtained by the sign of $c\left(\delta_{1}\right)$, the coefficient of critical normal form [25], and is computed via

$$
\begin{aligned}
c\left(\delta_{1}\right)= & \frac{1}{6}\langle p, C(q, q, q)\rangle \\
& -\frac{1}{2}\left\langle p, B\left(q,(A-I)^{-1} B(q, q)\right)\right\rangle \\
= & 2 \delta_{1}\left(2+\delta_{1}(b-d)\right)^{2} \neq 0 .
\end{aligned}
$$

The above discussion leads to the following result.

Theorem 4. If (17) holds and $\delta$ varies around the set $F B_{E_{1}}^{1}$, then system (4) passes through a flip bifurcation at fixed point $E_{1}(1,0)$. Moreover, the period-2 orbits that bifurcate from $E_{1}(1,0)$ are stable (resp., unstable) if $c\left(\delta_{1}\right)>0$ (resp., $c\left(\delta_{1}\right)<$ $0)$.

3.2. Flip and Neimark-Sacker Bifurcation at Fixed Point $E_{2}\left(x^{*}, y^{*}\right)$. We first discuss flip bifurcation of system (4) at fixed point $E_{2}$. We take parameter $(a, b, d, \delta)$ arbitrarily from $\mathrm{FB}_{E_{2}}^{1}$ (similarly for the case of $\mathrm{FB}_{E_{2}}^{2}$ ); then it follows that $P^{*}>$ 0 ; that is,

$$
\left(a_{1}-b_{2}\right)^{2}-4 a_{2} b_{1}>0
$$

Let

$$
\delta=\delta_{F}=\frac{N^{*}-\sqrt{P^{*}}}{M^{*}}
$$

or

$$
\delta=\delta_{F}=\frac{N^{*}+\sqrt{P^{*}}}{M^{*}}, \text { for the case of } \mathrm{FB}_{E_{2}}^{2} .
$$

Then the eigenvalues of positive fixed point $\left(x^{*}, y^{*}\right)$ are

$$
\begin{aligned}
& \lambda_{1}\left(\delta_{F}\right)=-1, \\
& \lambda_{2}\left(\delta_{F}\right)=3-\delta_{F} N^{*} .
\end{aligned}
$$

The condition $\left|\lambda_{2}\left(\delta_{F}\right)\right| \neq 1$ leads to

$$
\delta_{F} N^{*} \neq 2,4 .
$$

Using the transformation $\tilde{x}=x-x^{*}, \tilde{y}=y-y^{*}$ and writing $A(\delta)=J\left(x^{*}, y^{*}\right)$, we shift the fixed point $\left(x^{*}, y^{*}\right)$ of system (4) to the origin. After Taylor expansion, system (4) reduces to

$$
\left(\begin{array}{c}
\tilde{x} \\
\tilde{y}
\end{array}\right) \longrightarrow A(\delta)\left(\begin{array}{l}
\tilde{x} \\
\tilde{y}
\end{array}\right)+\left(\begin{array}{l}
F_{1}(\tilde{x}, \tilde{y}, \delta) \\
F_{2}(\tilde{x}, \tilde{y}, \delta)
\end{array}\right),
$$

where $X=(\tilde{x}, \tilde{y})^{T}$ and

$$
\begin{aligned}
& F_{1}(\tilde{x}, \tilde{y}, \delta)=\frac{1}{6}\left[-\frac{6 a \delta x^{* 2}}{\left(x^{*}+y^{*}\right)^{4}} \tilde{y}^{3}-\frac{6 a \delta y^{* 2}}{\left(x^{*}+y^{*}\right)^{4}} \tilde{x}^{3}\right. \\
& +\frac{2 a \delta\left(2 x^{*}-y^{*}\right) y^{*}}{\left(x^{*}+y^{*}\right)^{4}} 3 \tilde{x}^{2} \tilde{y} \\
& \left.+\frac{2 a \delta\left(x^{*}-2 y^{*}\right) x^{*}}{\left(x^{*}+y^{*}\right)^{4}} 3 \tilde{x} \tilde{y}^{2}\right]+\frac{1}{2}\left[\frac{2 a \delta x^{* 2}}{\left(x^{*}+y^{*}\right)^{3}} \tilde{y}^{2}\right. \\
& \left.+\left(-2 \delta+\frac{2 a \delta y^{* 2}}{\left(x^{*}+y^{*}\right)^{3}}\right) \tilde{x}^{2}-\frac{2 a \delta x^{*} y^{*}}{\left(x^{*}+y^{*}\right)^{3}} 2 \tilde{x} \tilde{y}\right] \\
& +O\left(\|X\|^{4}\right),
\end{aligned}
$$

$$
\begin{gathered}
F_{2}(\tilde{x}, \tilde{y}, \delta)=\frac{1}{6}\left[\frac{6 b \delta x^{* 2}}{\left(x^{*}+y^{*}\right)^{4}} \tilde{y}^{3}+\frac{6 b \delta y^{* 2}}{\left(x^{*}+y^{*}\right)^{4}} \tilde{x}^{3}\right. \\
-\frac{2 b \delta\left(2 x^{*}-y^{*}\right) y^{*}}{\left(x^{*}+y^{*}\right)^{4}} 3 \tilde{x}^{2} \tilde{y} \\
\left.+\frac{2 b \delta\left(x^{*}-2 y^{*}\right) x^{*}}{\left(x^{*}+y^{*}\right)^{4}} 3 \tilde{x} \tilde{y}^{2}\right]+\frac{1}{2}\left[-\frac{2 b \delta x^{* 2}}{\left(x^{*}+y^{*}\right)^{3}} \tilde{y}^{2}\right. \\
\left.-\frac{2 b \delta y^{* 2}}{\left(x^{*}+y^{*}\right)^{3}} \tilde{x}^{2}+\frac{2 b \delta x^{*} y^{*}}{\left(x^{*}+y^{*}\right)^{3}} 2 \tilde{x} \tilde{y}\right]+O\left(\|X\|^{4}\right) .
\end{gathered}
$$


It follows that

$$
\begin{aligned}
& B_{1}(x, y)=\left.\sum_{j, k=1}^{2} \frac{\delta^{2} F_{1}(\xi, \delta)}{\delta \xi_{j} \delta \xi_{k}}\right|_{\xi=0} x_{j} y_{k}=\frac{2 a \delta x^{* 2}}{\left(x^{*}+y^{*}\right)^{3}} \\
& \cdot x_{2} y_{2}+\left(-2 \delta+\frac{2 a \delta y^{* 2}}{\left(x^{*}+y^{*}\right)^{3}}\right) x_{1} y_{1} \\
& -\frac{2 a \delta x^{*} y^{*}}{\left(x^{*}+y^{*}\right)^{3}}\left(x_{1} y_{2}+x_{2} y_{1}\right) \\
& B_{2}(x, y)=\left.\sum_{j, k=1}^{2} \frac{\delta^{2} F_{2}(\xi, \delta)}{\delta \xi_{j} \delta \xi_{k}}\right|_{\xi=0} x_{j} y_{k}=-\frac{2 b \delta x^{* 2}}{\left(x^{*}+y^{*}\right)^{3}} \\
& x_{2} y_{2}-\frac{2 b \delta y^{* 2}}{\left(x^{*}+y^{*}\right)^{3}} x_{1} y_{1}+\frac{2 b \delta x^{*} y^{*}}{\left(x^{*}+y^{*}\right)^{3}}\left(x_{1} y_{2}\right. \\
& \left.+x_{2} y_{1}\right) \text {, } \\
& C_{1}(x, y, u)=\left.\sum_{j, k, l=1}^{2} \frac{\delta^{2} F_{1}(\xi, \delta)}{\delta \xi_{j} \delta \xi_{k} \delta \xi_{l}}\right|_{\xi=0} x_{j} y_{k} u_{l} \\
& =-\frac{6 a \delta x^{* 2}}{\left(x^{*}+y^{*}\right)^{4}} x_{2} y_{2} u_{2}-\frac{6 a \delta y^{* 2}}{\left(x^{*}+y^{*}\right)^{4}} x_{1} y_{1} u_{1} \\
& +\frac{2 a \delta\left(2 x^{*}-y^{*}\right) y^{*}}{\left(x^{*}+y^{*}\right)^{4}}\left(x_{1} y_{1} u_{2}+x_{1} y_{2} u_{1}+x_{2} y_{1} u_{1}\right. \\
& \left.+x_{2} y_{1} u_{2}+x_{1} y_{2} u_{2}+x_{2} y_{2} u_{1}\right) \text {, } \\
& C_{2}(x, y, u)=\left.\sum_{j, k, l=1}^{2} \frac{\delta^{2} F_{2}(\xi, \delta)}{\delta \xi_{j} \delta \xi_{k} \delta \xi_{l}}\right|_{\xi=0} x_{j} y_{k} u_{l} \\
& =\frac{6 b \delta x^{* 2}}{\left(x^{*}+y^{*}\right)^{4}} x_{2} y_{2} u_{2}+\frac{6 b \delta y^{* 2}}{\left(x^{*}+y^{*}\right)^{4}} x_{1} y_{1} u_{1} \\
& -\frac{2 b \delta\left(x^{*}-2 y^{*}\right) x^{*}}{\left(x^{*}+y^{*}\right)^{4}}\left(x_{1} y_{1} u_{2}+x_{1} y_{2} u_{1}+x_{2} y_{1} u_{1}\right. \\
& \left.+x_{2} y_{1} u_{2}+x_{1} y_{2} u_{2}+x_{2} y_{2} u_{1}\right) \text {, }
\end{aligned}
$$

and $\delta=\delta_{F}$.

Therefore, we get the following symmetric multilinear vector functions of $x, y, u \in \mathbb{R}^{2}: B(x, y)=\left(\begin{array}{l}B_{1}(x, y) \\ B_{2}(x, y)\end{array}\right)$ and $C(x, y, u)=\left(\begin{array}{l}C_{1}(x, y, u) \\ C_{2}(x, y, u)\end{array}\right)$.

Let $p, q \in \mathbb{R}^{2}$ be two eigenvectors (left and right) of $A$ associated with eigenvalue $\lambda_{1}\left(\delta_{F}\right)=-1$, respectively. Then $A\left(\delta_{F}\right) q=-q$ and $A^{T}\left(\delta_{F}\right) p=-p$. After some algebraic calculation, we get

$$
\begin{aligned}
& q \sim\left(-2-\delta_{F} b_{2}, \delta_{F} a_{2}\right)^{T}, \\
& p \sim\left(-2-\delta_{F} b_{2},-\delta_{F} b_{1}\right)^{T} .
\end{aligned}
$$

We set $p=\gamma_{1}\left(-2-\delta_{F} b_{2},-\delta_{F} b_{1}\right)^{T}$, where

$$
\gamma_{1}=\frac{1}{\left(-2-\delta_{F} b_{2}\right)^{2}-\delta_{F}^{2} a_{2} b_{1}} \text {. }
$$

Then by the standard scalar product in $\mathbb{R}^{2}$ defined by $\langle p, q\rangle=p_{1} q_{1}+p_{2} q_{2}$, it is obvious to see that $\langle p, q\rangle=1$. The direction of the flip bifurcation can be obtained by the sign of $c\left(\delta_{F}\right)$, the coefficient of critical normal form [25], and is computed via

$$
\begin{aligned}
c\left(\delta_{F}\right)= & \frac{1}{6}\langle p, C(q, q, q)\rangle \\
& -\frac{1}{2}\left\langle p, B\left(q,(A-I)^{-1} B(q, q)\right)\right\rangle .
\end{aligned}
$$

The above discussion leads to the following result.

Theorem 5. If (29) holds, $c\left(\delta_{F}\right) \neq 0$, and $\delta$ varies around the set $\mathrm{FB}_{E_{2}}^{1}$, then system (4) passes through a flip bifurcation at fixed point $E_{2}\left(x^{*}, y^{*}\right)$. Moreover, the period-2 orbits that bifurcate from $E_{2}\left(x^{*}, y^{*}\right)$ are stable (resp., unstable) if $c\left(\delta_{F}\right)>$ 0 (resp., $\left.c\left(\delta_{F}\right)<0\right)$. (4).

We next discuss a Neimark-Sacker bifurcation of system

We first take parameter $(a, b, d, \delta)$ arbitrarily from $\mathrm{NSB}_{E_{2}}$ and consider system (4) at fixed point $E_{2}\left(x^{*}, y^{*}\right)$.

Since the parameters belong to $\mathrm{NSB}_{E_{2}}$, the complex eigenvalues of (6) are

$$
\lambda, \bar{\lambda}=\frac{\operatorname{tr} J}{2} \pm \frac{i}{2} \sqrt{4 \operatorname{det} J-(\operatorname{tr} J)^{2}},
$$

where the condition $(\operatorname{tr} J)^{2}-4 \operatorname{det} J<0$ leads to $\left(a_{1}-b_{2}\right)^{2}-$ $4 a_{2} b_{1}<0$. Let

$$
\delta=\delta_{\mathrm{NS}}=\frac{N^{*}}{M^{*}}
$$

then $\operatorname{det} J\left(\delta_{\mathrm{NS}}\right)=1$ and

$$
\begin{aligned}
|\lambda| & =\sqrt{\operatorname{det} J(\delta)}, \\
\left.\frac{d|\lambda(\delta)|}{d \delta}\right|_{\delta=\delta_{\mathrm{NS}}} & =\frac{N^{*}}{2} \neq 0 .
\end{aligned}
$$

Moreover, if $\operatorname{tr} J\left(\delta_{\mathrm{NS}}\right) \neq 0,-1$, then $\delta_{\mathrm{NS}} N^{*} \neq 2,3$ which leads to

$$
\lambda^{k}\left(\delta_{\mathrm{NS}}\right) \neq 1 \quad \text { for } k=1,2,3,4
$$

Let $q, p \in \mathbb{C}^{2}$ be eigenvectors of $A\left(\delta_{\mathrm{NS}}\right)$ and $A^{T}\left(\delta_{\mathrm{NS}}\right)$ corresponding to the eigenvalues $\lambda\left(\delta_{\mathrm{NS}}\right)$ and $\bar{\lambda}\left(\delta_{\mathrm{NS}}\right)$ such that

$$
\begin{gathered}
A\left(\delta_{\mathrm{NS}}\right) q=\lambda\left(\delta_{\mathrm{NS}}\right) q, \\
A\left(\delta_{\mathrm{NS}}\right) \bar{q}=\bar{\lambda}\left(\delta_{\mathrm{NS}}\right) \bar{q}, \\
A^{T}\left(\delta_{\mathrm{NS}}\right) p=\bar{\lambda}\left(\delta_{\mathrm{NS}}\right) p, \\
A^{T}\left(\delta_{\mathrm{NS}}\right) \bar{p}=\lambda\left(\delta_{\mathrm{NS}}\right) \bar{p} .
\end{gathered}
$$


Then by algebraic calculation, we obtain

$$
\begin{aligned}
& q \sim\left(1+\delta_{\mathrm{NS}} b_{2}-\lambda,-\delta_{\mathrm{NS}} a_{2}\right)^{T}, \\
& p \sim\left(1+\delta_{\mathrm{NS}} b_{2}-\bar{\lambda}, \delta_{\mathrm{NS}} b_{1}\right)^{T} .
\end{aligned}
$$

We set $p=\gamma_{2}\left(1+\delta_{\mathrm{NS}} b_{2}-\bar{\lambda}, \delta_{\mathrm{NS}} b_{1}\right)^{T}$, where

$$
\gamma_{2}=\frac{1}{\left(1+\delta_{\mathrm{NS}} b_{2}-\bar{\lambda}\right)^{2}-\delta_{\mathrm{NS}}^{2} a_{2} b_{1}} .
$$

Then it is obvious that $\langle p, q\rangle=1$, where $\langle p, q\rangle=\overline{p_{1}} q_{2}+$ $\overline{p_{2}} q_{1}$ for $p, q \in \mathbb{C}^{2}$. We can decompose vector $X \in \mathbb{R}^{2}$ as $X=z q+\bar{z} q$, for $\delta$ close to $\delta_{\mathrm{NS}}$ and $z \in \mathbb{C}$. Obviously, $z=$ $\langle p, X\rangle$. Thus, we obtain the following transformed form of system (30) for $|\delta|$ near $\delta_{\mathrm{NS}}$ :

$$
z \longmapsto \lambda(\delta) z+g(z, \bar{z}, \delta),
$$

where $\lambda(\delta)=(1+\varphi(\delta)) e^{i \theta(\delta)}$ with $\varphi\left(\delta_{\mathrm{NS}}\right)=0$ and $g(z, \bar{z}, \delta)$ is a smooth complex-valued function. Taylor expression of $g$ with respect to $(z, \bar{z})$ yields

$$
\begin{aligned}
g(z, \bar{z}, \delta)=\sum_{k+l \geq 2} \frac{1}{k ! l !} g_{k l}(\delta) z^{k} \bar{z}^{l} & \\
& \text { with } g_{k l} \in \mathbb{C}, k, l=0,1, \ldots
\end{aligned}
$$

According to multilinear symmetric vector functions, we can express the coefficients $g_{k l}$ as follows:

$$
\begin{aligned}
& g_{20}\left(\delta_{\mathrm{NS}}\right)=\langle p, B(q, q)\rangle, \\
& g_{11}\left(\delta_{\mathrm{NS}}\right)=\langle p, B(q, \bar{q})\rangle, \\
& g_{02}\left(\delta_{\mathrm{NS}}\right)=\langle p, B(\bar{q}, \bar{q})\rangle, \\
& g_{21}\left(\delta_{\mathrm{NS}}\right)=\langle p, C(q, q, \bar{q})\rangle .
\end{aligned}
$$

We calculate the coefficient $a\left(\delta_{\mathrm{NS}}\right)$ by the following formula to determine the direction in which invariant closed curve appears.

$$
\begin{aligned}
a\left(\delta_{\mathrm{NS}}\right)= & \operatorname{Re}\left(\frac{e^{-i \theta\left(\delta_{\mathrm{NS}}\right)} g_{21}}{2}\right) \\
& -\operatorname{Re}\left(\frac{\left(1-2 e^{i \theta\left(\delta_{\mathrm{NS}}\right)}\right) e^{-2 i \theta\left(\delta_{\mathrm{NS}}\right)}}{2\left(1-e^{i \theta\left(\delta_{\mathrm{NS}}\right)}\right)} g_{20} g_{11}\right) \\
& -\frac{1}{2}\left|g_{11}\right|^{2}-\frac{1}{4}\left|g_{02}\right|^{2},
\end{aligned}
$$

where $e^{i \theta\left(\delta_{\mathrm{NS}}\right)}=\lambda\left(\delta_{\mathrm{NS}}\right)$.

It is clear that two conditions (41) and (42) known as transversal and nondegenerate hold well for system (4). Therefore, we have the following result.

Theorem 6. If $a\left(\delta_{\mathrm{NS}}\right) \neq 0$ and $\delta$ changes its value around $\mathrm{NSB}_{E_{2}}$, then system (4) passes through a Neimark-Sacker bifurcation at fixed point $E_{2}$. Moreover, if $a\left(\delta_{\mathrm{NS}}\right)<0$ (resp., $>0$ ), then there exists an attracting (resp., repelling) invariant closed curve which bifurcates from $E_{2}$.
TABLE 1: Set of parameter values.

\begin{tabular}{lcc}
\hline & Varying parameter & Fixed parameters \\
\hline Case (i) & $1.8 \leq \delta \leq 3$ & $a=1.0, b=0.5, d=0.6$ \\
Case (ii) & $2.05 \leq \delta \leq 3.2$ & $a=1.67, b=1.0, d=0.6$ \\
Case (iii) & $2.05 \leq \delta \leq 3.2,1.6 \leq a \leq 1.7$ & $b=1.0, d=0.6$ \\
\hline
\end{tabular}

\section{Numerical Simulations}

Here, diagrams for bifurcation, phase portraits, Lyapunov exponents, and fractal dimension of system (4) will be presented by performing numerical simulation to validate our analytical results. The parameter $\delta$ is chosen as a bifurcation parameter to see the effect of ratio-dependent functional response in the system. We restrict our attention in the case of NS bifurcation only.

Now we consider bifurcation parameters in the following cases.

For case (i): by taking parameters as in Table 1, we see that, at the fixed point $(1,0)$, a flip bifurcation occurs at $\delta=\delta_{1}=2$ and it shows $c\left(\delta_{1}\right)=12.96>0$. This confirms Theorem 4 .

Figure 2(a) displays the bifurcation diagram of system (4). This illustrates that stability of fixed point $E_{1}$ happens for $\delta<2$, bifurcation occurs at $\delta=2$, and there appears period doubling bifurcation if $\delta>2$. Figure 2(b) results in the maximum Lyapunov exponents relating bifurcation in Figure 2(a) confirming the parametric space in which the behaviors of system (4) are chaotic or stable period window.

For case (ii): by taking parameters as in Table 1, we see that, at the fixed point $(0.332,0.221333)$, a NS bifurcation emerges at $\delta=\delta_{\mathrm{NS}}=2.14859$ and it shows $a\left(\delta_{\mathrm{NS}}\right)=$ $-0.630982<0$. This confirms Theorem 6 .

Figures 3(a) and 3(b) display the bifurcation diagrams of system (4). This illustrates that stability of fixed point $E_{2}$ happens for $\delta<2.14859$, bifurcation occurs at $\delta=2.14859$, and there appears attracting invariant circle if $\delta>2.14859$. Figure 3(c) results in the maximum Lyapunov exponents relating bifurcation in Figures 3(a) and 3(b) confirming the parametric space in which the behaviors of system (4) are chaotic or stable period window. Figure $3(d)$ is local amplification of Figure 3(a) for $\delta \in[2.6503,2.999]$.

The phase portraits of bifurcation diagrams related to Figures 3(a) and 3(b) for various values of $\delta$ are plotted in Figure 4, which clearly illustrates the act of smooth invariant circle and how it bifurcates from the stable fixed point. We observe that as $\delta$ increases, the invariant circle suddenly disappears and periods 9,26 , and 19 orbits appear at $\delta=$ $2.7,2.902,2.93$, respectively. We also see that for $\delta=3.2$, there exists a fully developed chaos in system (4).

For case (iii): the dynamics of map (4) can change when more parameters vary. The diagrams of bifurcation of map (4) for control parameters $\delta \in[2.05,3.2], a \in[1.6,1.7]$, respectively, and the fixing remaining parameters as in case (ii) are disposed in Figures 5(a) and 5(b). The 3D maximum Lyapunov exponent for two control parameters $\delta$ and $a$ is plotted in Figure $5(\mathrm{c})$ and its $2 \mathrm{D}$ projection onto $(\delta, a)$ is shown in Figure 5(d). It is easy to find values of control parameters for which the dynamics of map (4) is in chaotic, 


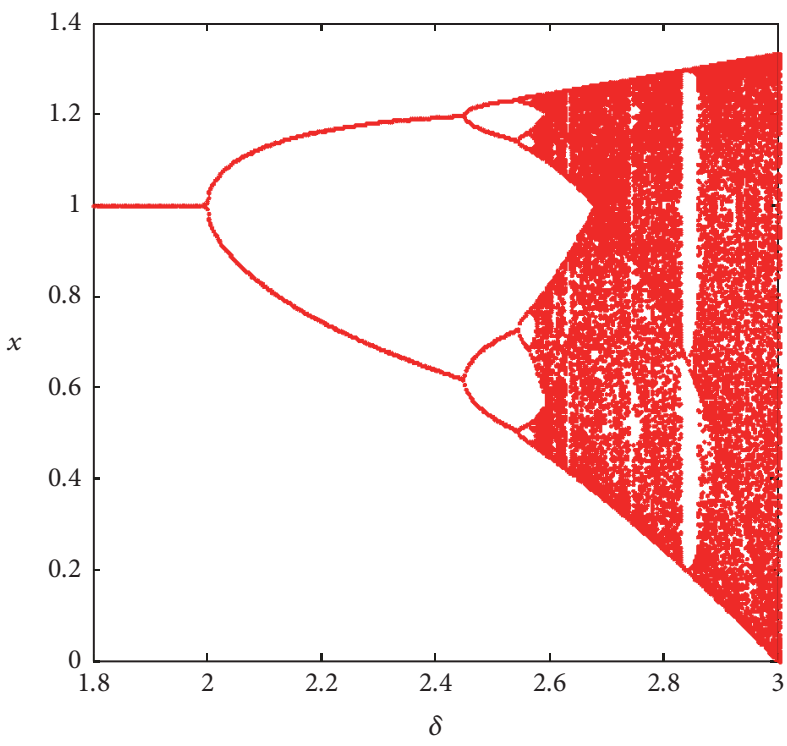

(a)

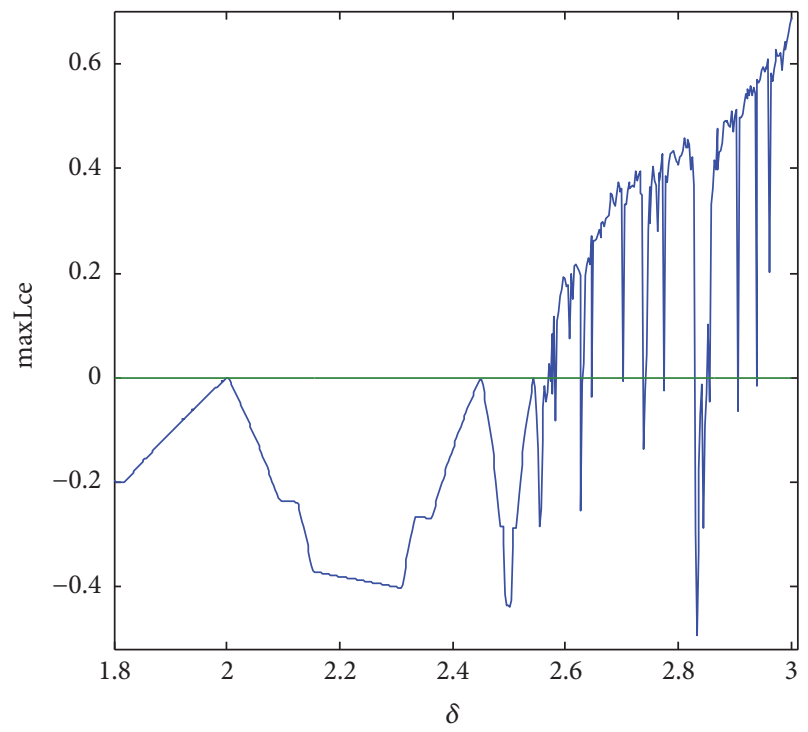

(b)

FIGURE 2: Flip bifurcation and Lyapunov exponent of system (4). (a) Bifurcation for prey and (b) maximum Lyapunov exponents related to (a). Initial value: $\left(x_{0}, y_{0}\right)=(0.98,0.1)$.

periodic, or nonchaotic status. Obviously, there are chaotic dynamics for $\delta=3.1492$ and $a=1.67$ and nonchaotic dynamics for $\delta=2.7$ and $a=1.67$ (see Figure 4), which are compatible with the signs of Lyapunov exponents in Figure 5(c). This exhibits that as the parameter $a$ increases, the map (4) shows more chaotic dynamics.

4.1. Initial Perturbation. Sensitivity to initial values of a system demonstrates that at the beginning the two trajectories are arbitrarily closely overlapped but a significant difference builds up for future trajectories. For numerical simulation, two perturbed trajectories for state variable $x$ of system (4) against the number of iterations with initial points $\left(x_{0}, y_{0}\right)$ and $\left(x_{0}+0.001, y_{0}\right)$ are generated and plotted in Figure 6(a). The difference (error) between two trajectories is computed by $\Delta x=x_{1}-x_{2}$ and presented in Figure 6(b), where $x_{1}$ and $x_{2}$ are solution trajectories of system (4) without and with initial perturbation. We take 1000 iterations for each initial value. From Figure 6, it is clear that the error is negligible initially but it increases for trajectories in future time. Therefore, a slightly initial perturbation may lead to complex dynamic behavior of the system.

4.2. Fractal Dimension. The fractal dimension (FD) $[29,30]$ which characterizes strange attractors of a map is defined by

$$
d_{L}=j+\frac{\sum_{i=1}^{j} \lambda_{i}}{\left|\lambda_{j}\right|},
$$

where $\lambda_{1}, \lambda_{2}, \ldots, \lambda_{n}$ are Lyapunov exponents and $j$ is the largest integer such that $\sum_{i=1}^{j} \lambda_{i} \geq 0$ and $\sum_{i=1}^{j+1} \lambda_{i}<0$. For our two-dimensional map (4), the fractal dimension takes the form

$$
d_{L}=1+\frac{\lambda_{1}}{\left|\lambda_{2}\right|}, \quad \lambda_{1}>0>\lambda_{2}
$$

Taking parameter values given as in case (i) and by computer simulation, two Lyapunov exponents are $\lambda_{1} \approx$ 0.0281 and $\lambda_{2} \approx-0.0741$ for $\delta=2.9815$ and $\lambda_{1} \approx 0.0832$ and $\lambda_{2} \approx-0.0950$ for $\delta=3.1494$. So the fractal dimensions of map (4) are

$$
\begin{aligned}
& d_{L} \approx 1+\frac{0.0281}{0.0741}=1.3793 \quad \text { for } \delta=2.9815, \\
& d_{L} \approx 1+\frac{0.0832}{0.0950}=1.8759 \quad \text { for } \delta=3.1494 .
\end{aligned}
$$

The strange attractors given in Figure 4 are consistent with the delta values' corresponding fractal dimension and this illustrates that the ratio-dependent discrete predator-prey system (4) has a very complex dynamic behavior as the parameter $\delta$ increases. We refer to Figure 7 to understand the system dynamics using fractal dimension.

\section{Chaos Control}

We shall apply a state feedback control method $[24,31]$ in order to stabilize chaos of system (4) at the state of unstable trajectories. By adding a feedback control law as the control force $u_{n}$ to system (4), the controlled form of (4) becomes

$$
\begin{aligned}
& x_{n+1}=x_{n}+\delta\left[x_{n}\left(1-x_{n}\right)-\frac{a x_{n} y_{n}}{y_{n}+x_{n}}\right]+u_{n}, \\
& y_{n+1}=y_{n}+\delta\left[-d y_{n}+\frac{b x_{n} y_{n}}{y_{n}+x_{n}}\right]
\end{aligned}
$$




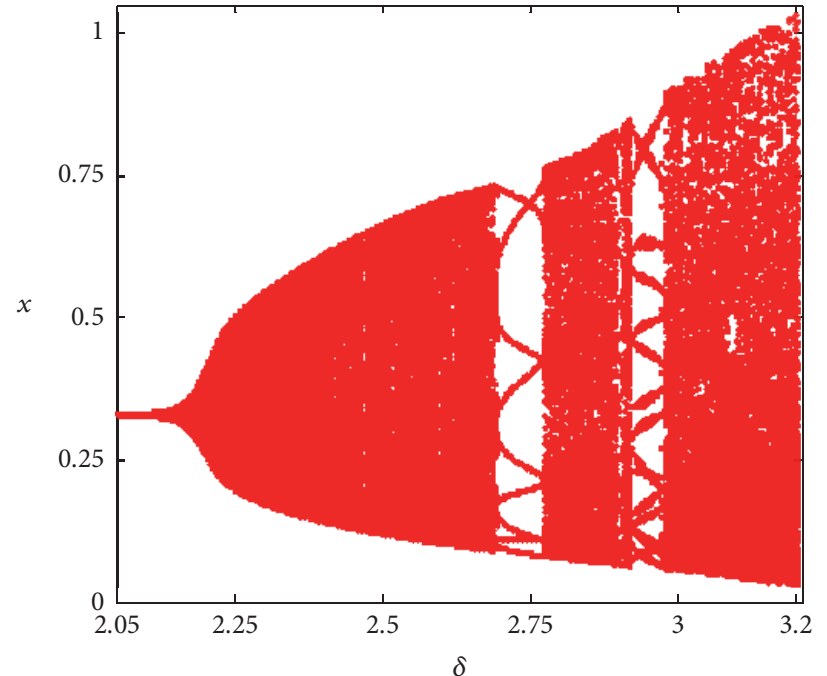

(a)

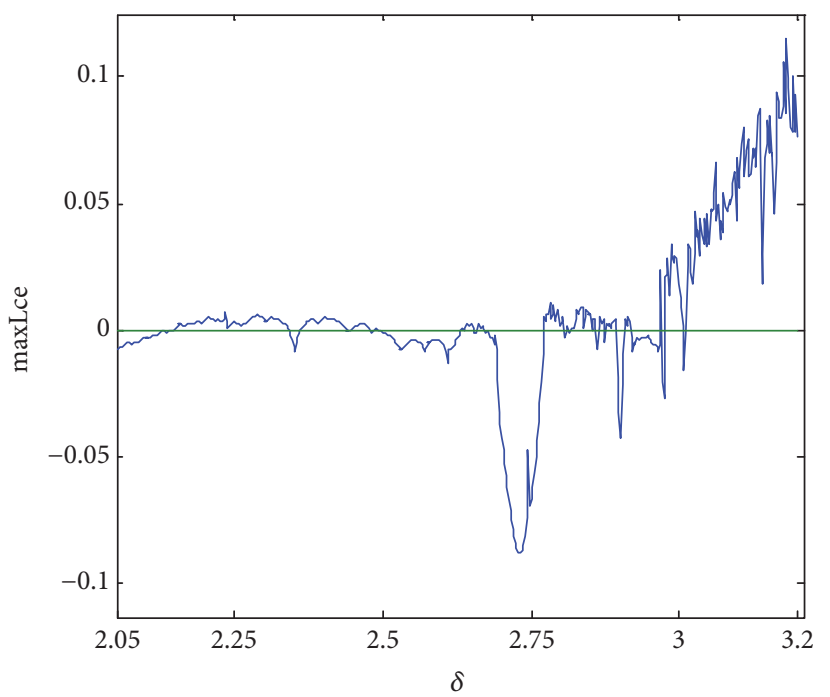

(c)

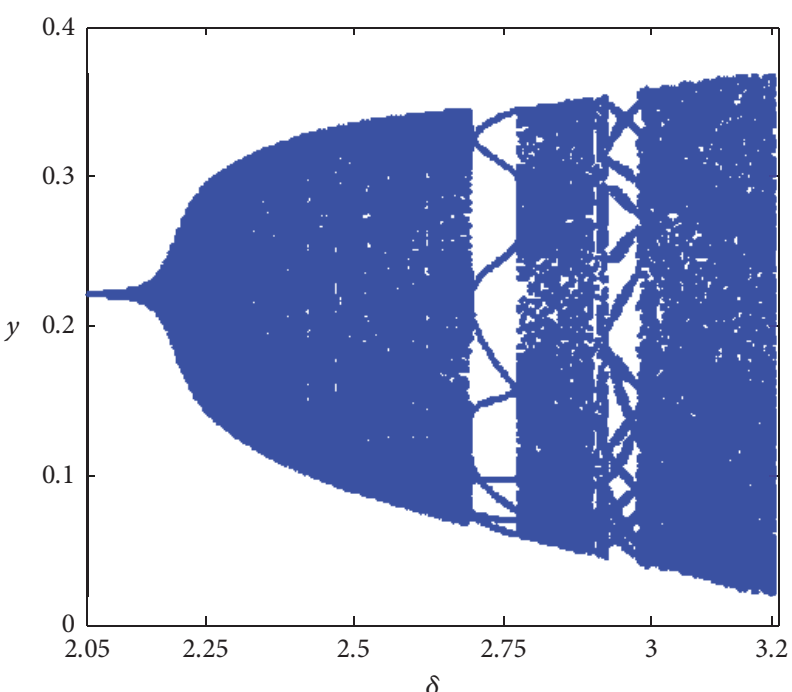

(b)

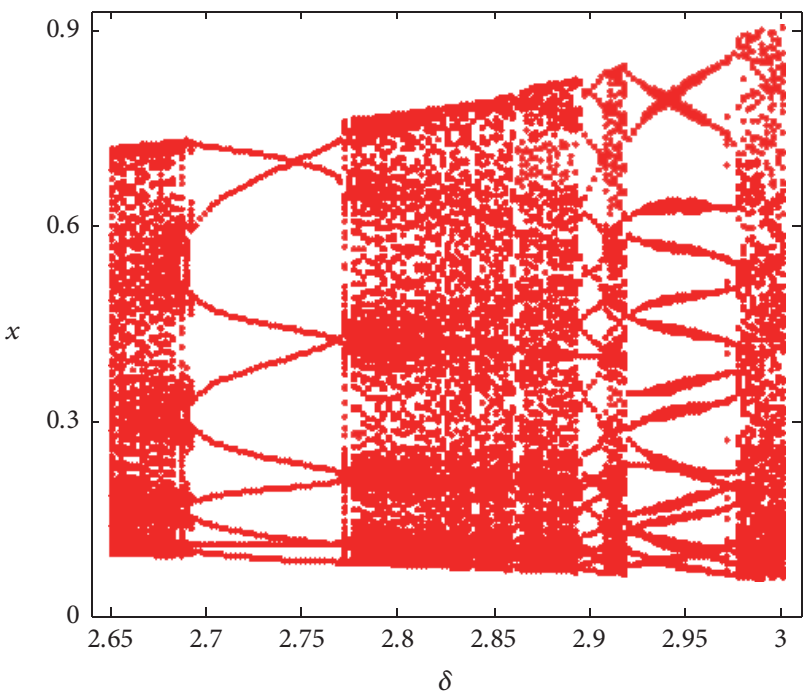

(d)

FIGURE 3: Neimark-Sacker bifurcation and Lyapunov exponent of system (4). (a) Bifurcation for prey, (b) bifurcation for predator, (c) maximum Lyapunov exponents related to (a-b), and (d) local amplification related to (a) for $\delta \in[2.6503,2.999]$. Initial value: $\left(x_{0}, y_{0}\right)=$ $(0.324,0.2163)$.

$$
u_{n}=-k_{1}\left(x_{n}-x^{*}\right)-k_{2}\left(y_{n}-y^{*}\right)
$$

where the feedback gains are denoted by $k_{1}$ and $k_{2}$ and $\left(x^{*}, y^{*}\right)$ represent positive fixed point of $(4)$.

The Jacobian matrix $J_{c}$ of the controlled system (53) is given by

$$
J_{c}\left(x^{*}, y^{*}\right)=\left(\begin{array}{cc}
a_{11}-k_{1} & a_{12}-k_{2} \\
a_{21} & a_{22}
\end{array}\right) \text {, }
$$

where $a_{11}=1+\delta a_{1}, a_{12}=-\delta b_{1}, a_{21}=\delta a_{2}, a_{22}=1+\delta b_{2}$, and $a_{1}, b_{1}, a_{2}$, and $b_{2}$ are determined by (7). The characteristic equation of matrix $J_{c}$ is

$$
\lambda^{2}-\left(\operatorname{tr} J_{c}\right) \lambda+\operatorname{det} J_{c}=0
$$

where $\operatorname{tr} J_{c}=a_{11}+a_{22}-k_{1}$ and det $J_{c}=a_{22}\left(a_{11}-k_{1}\right)-a_{21}\left(a_{12}-\right.$ $\left.k_{2}\right)$. Let $\lambda_{1}$ and $\lambda_{2}$ be the roots of (56), that is, eigenvalues of (55). Then

$$
\begin{aligned}
\lambda_{1}+\lambda_{2} & =\operatorname{tr} J_{c}, \\
\lambda_{1} \lambda_{2} & =\operatorname{det} J_{c} .
\end{aligned}
$$

The solution of the equations $\lambda_{1}= \pm 1$ and $\lambda_{1} \lambda_{2}=1$ determines the lines of marginal stability. These conditions confirm that $\left|\lambda_{1,2}\right|<1$. Assume that $\lambda_{1} \lambda_{2}=1$; then from (58) we have $\operatorname{det} J_{c}=1$; that is,

$$
l_{1}: a_{22} k_{1}-a_{21} k_{2}=a_{11} a_{22}-a_{12} a_{21}-1 \text {. }
$$




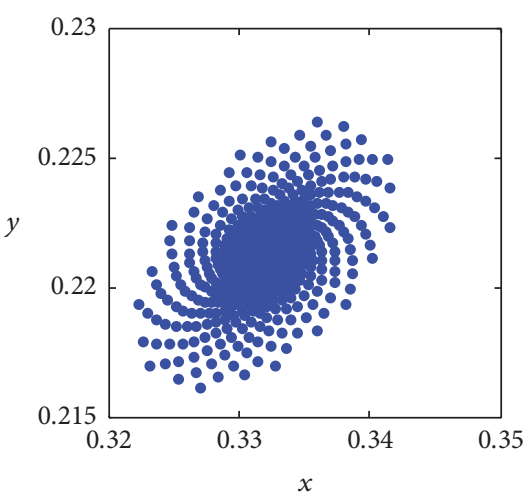

$\delta=2.103$

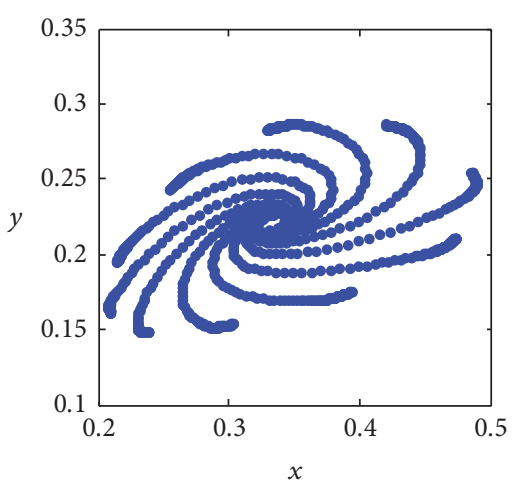

$\delta=2.236$

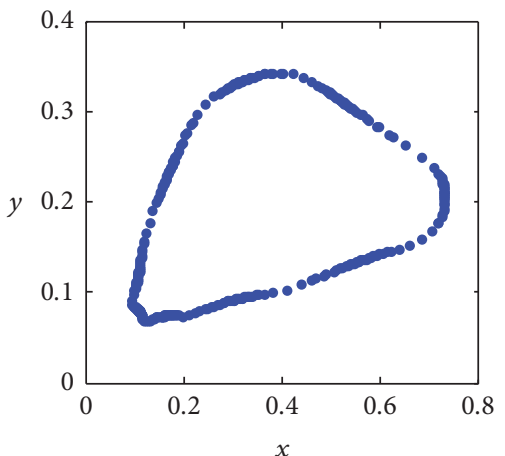

$\delta=2.686$

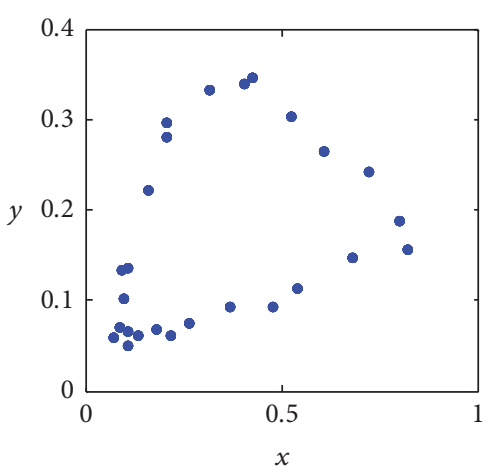

$\delta=2.902$

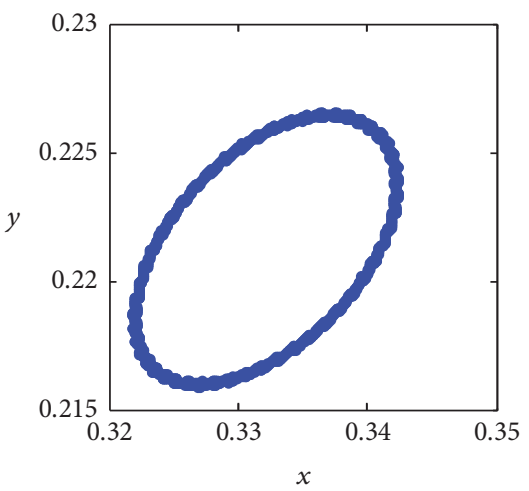

$\delta=2.14859$

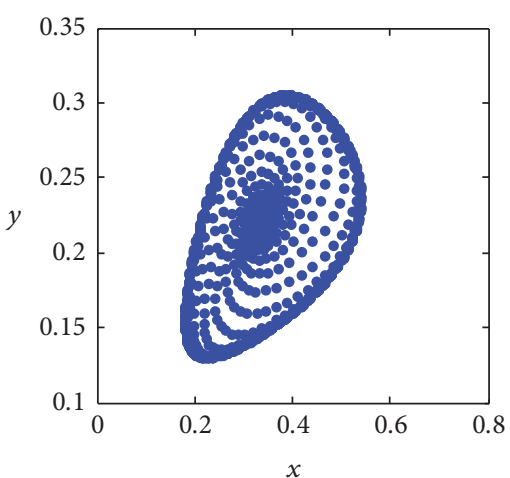

$\delta=2.29$

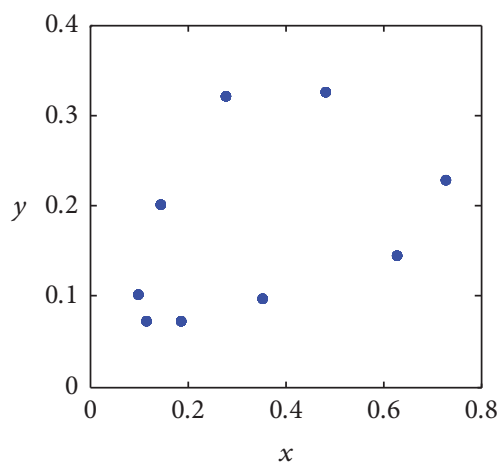

$\delta=2.7$

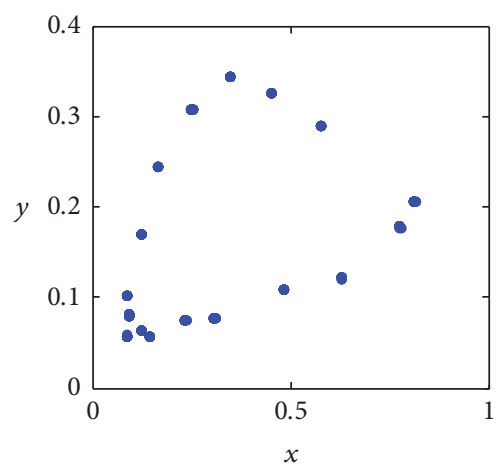

$\delta=2.93$

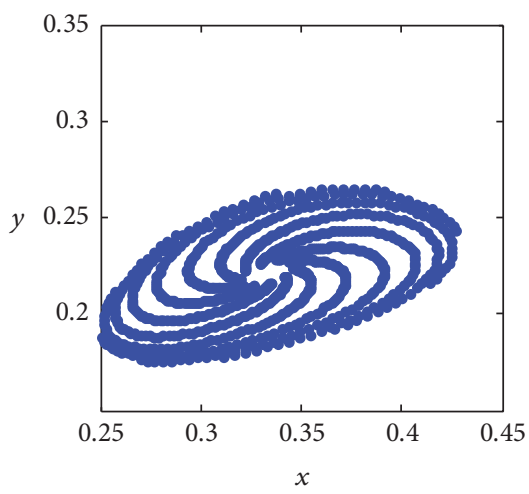

$\delta=2.186$

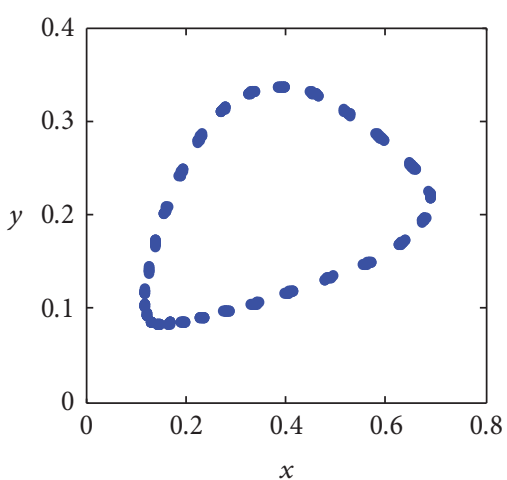

$\delta=2.55$

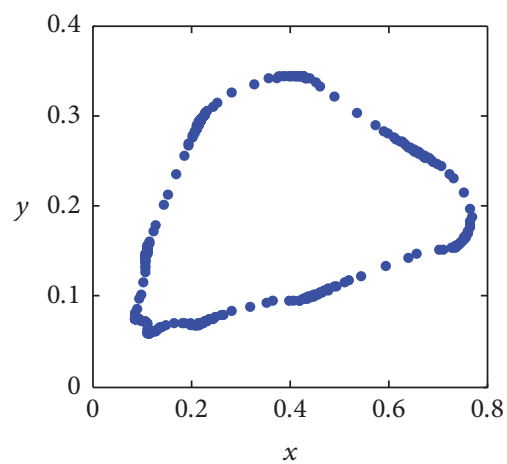

$\delta=2.78$

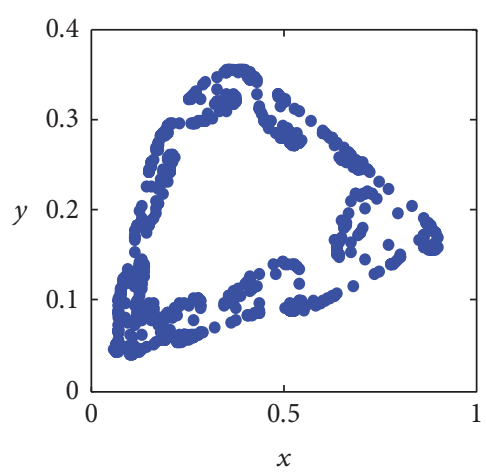

$\delta=2.9815$

FIgure 4: Continued. 


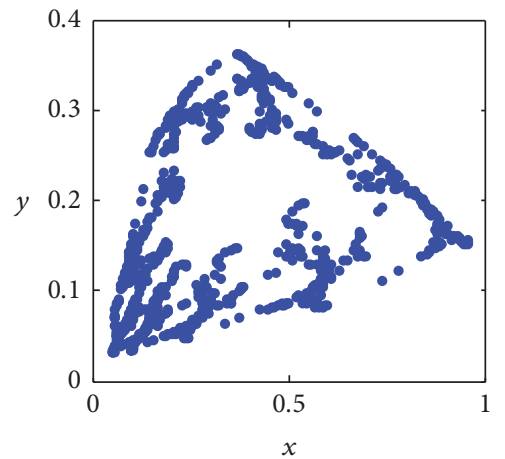

$\delta=3.07$

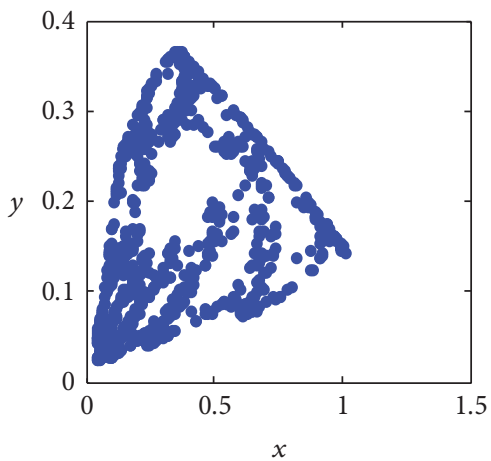

$\delta=3.1494$

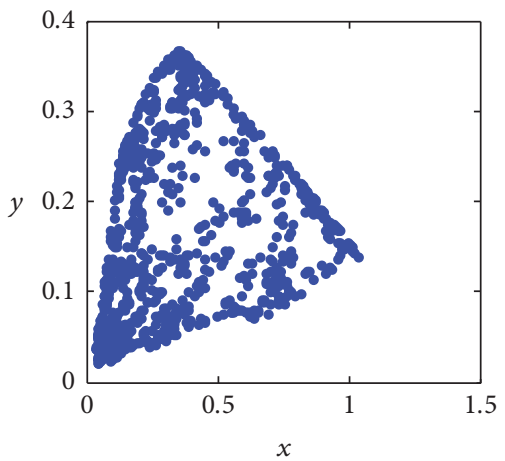

$\delta=3.2$

FIGURE 4: Phase portraits of bifurcation diagrams Figures 3(a) and 3(b) for several values of $\delta$.

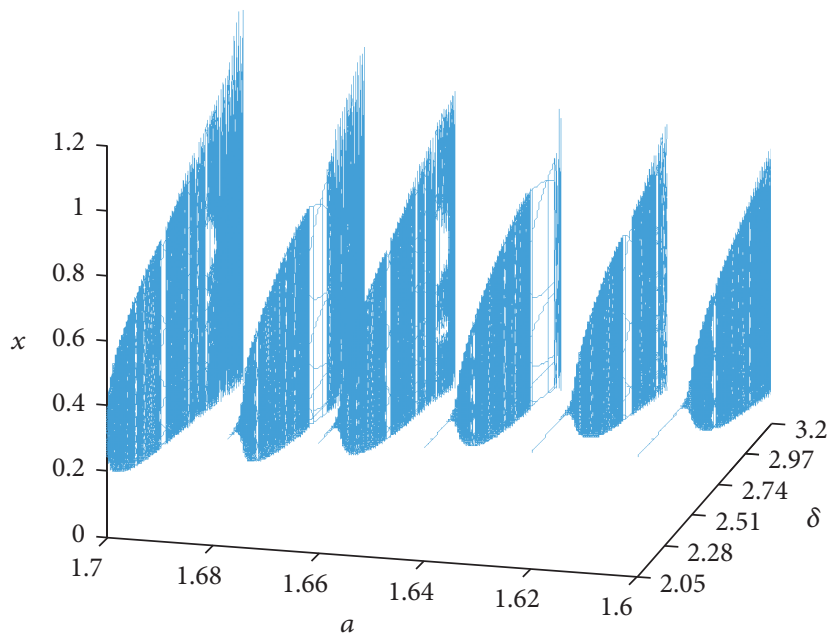

(a)

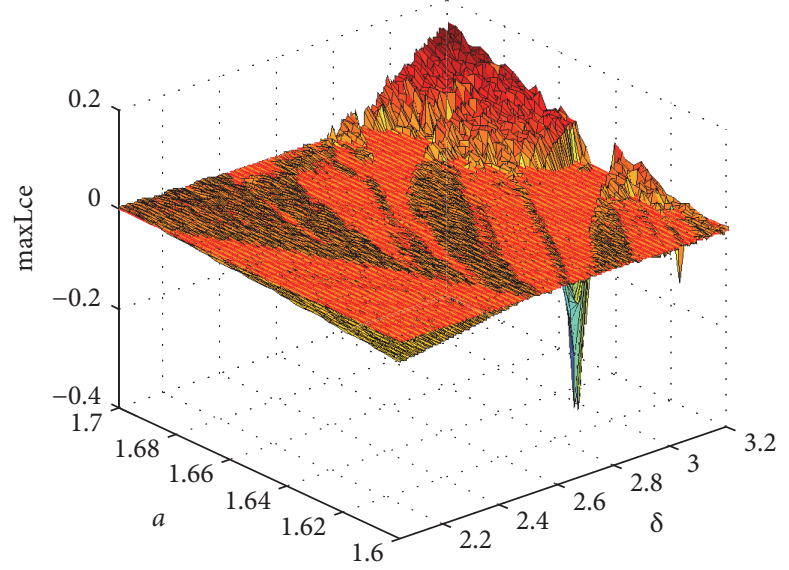

(c)

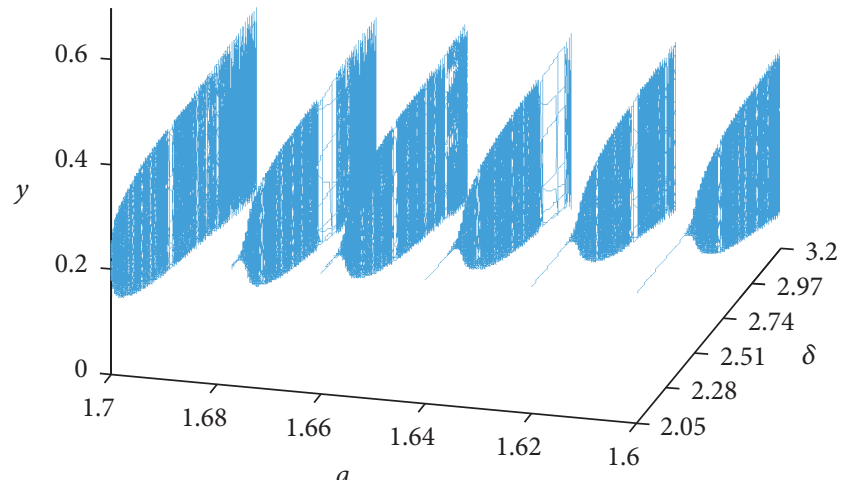

(b)

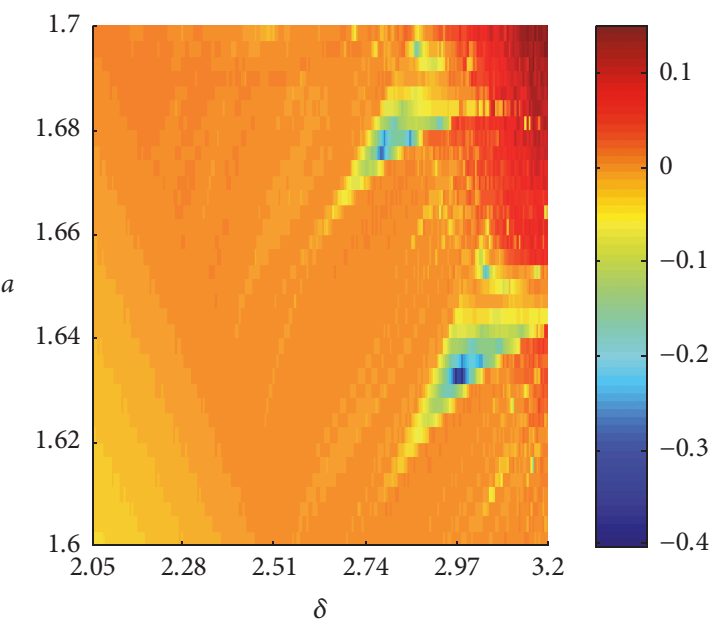

(d)

FIGURE 5: Numerical diagnostic of system (4) for control parameters $\delta$ and $a$. (a-b) Bifurcation for prey and predator covering $\delta \in[2.05,3.2]$, $a \in[1.6,1.7]$, and $b=1.0, d=0.6$ (c) The 3D view of maximum Lyapunov exponents related to (a-b). (d) The $2 \mathrm{D}$ projection onto $(\delta, a)$. Initial value: $\left(x_{0}, y_{0}\right)=(0.324,0.2163)$. 


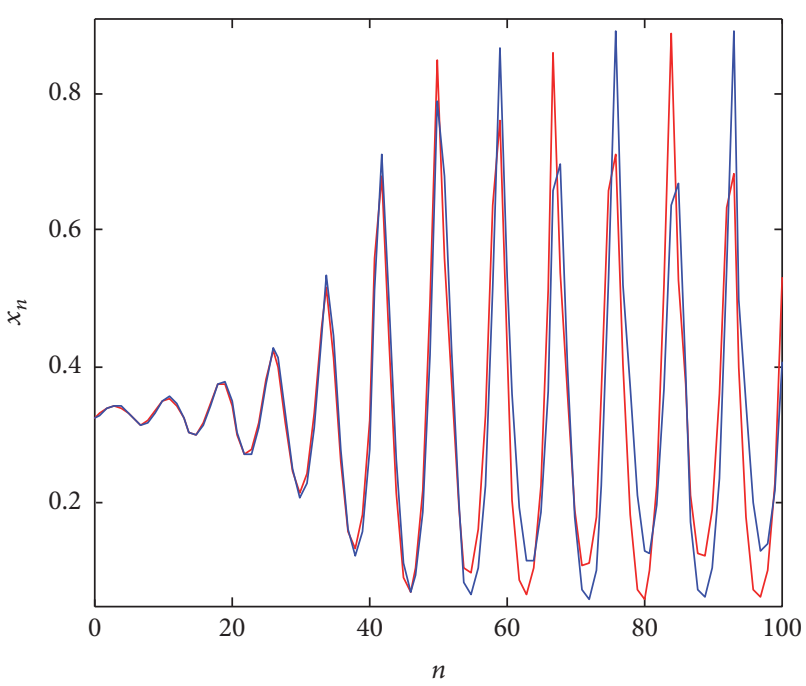

(a)

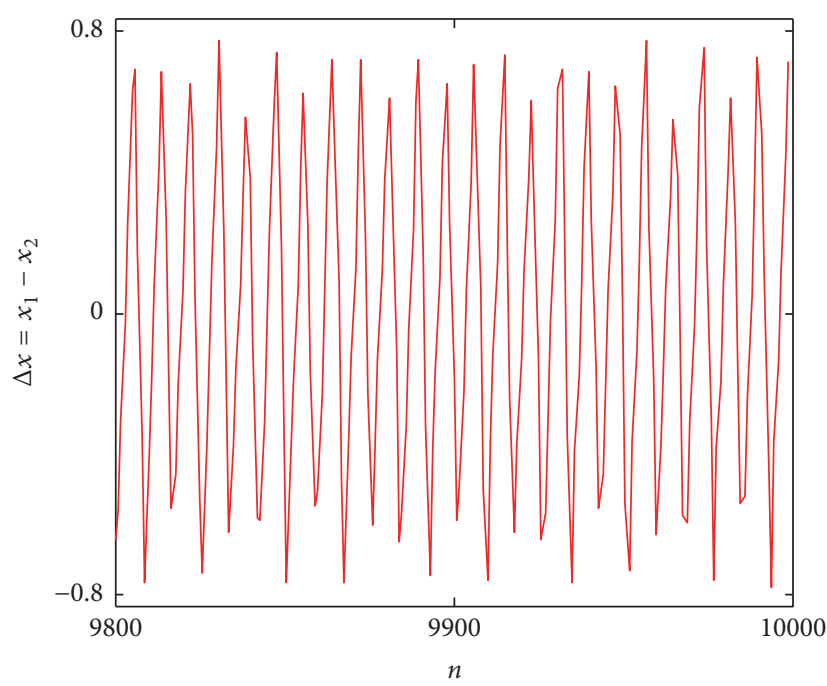

(b)

FIGURE 6: Initial perturbation for system (4). (a) Trajectories for state variable $x$, blue for $\left(x_{0}, y_{0}\right)$; red for $\left(x_{0}+0.001, y_{0}\right)$. (b) Error diagnostic of the two trajectories. Parameters: $a=1.67, b=1.0, d=0.6, \delta=2.98$. Initial value: $\left(x_{0}, y_{0}\right)=(0.324,0.21633)$.

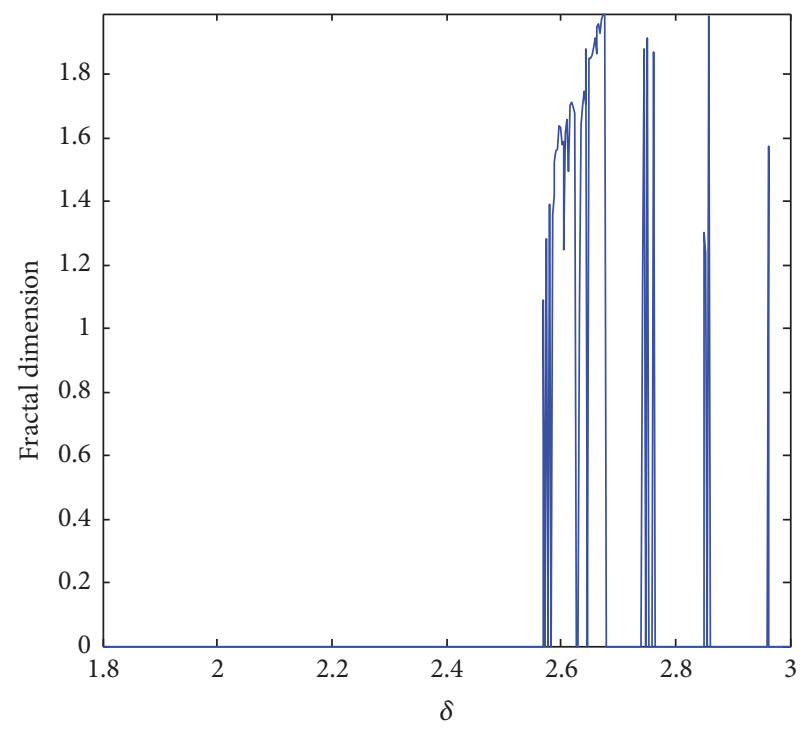

(a)

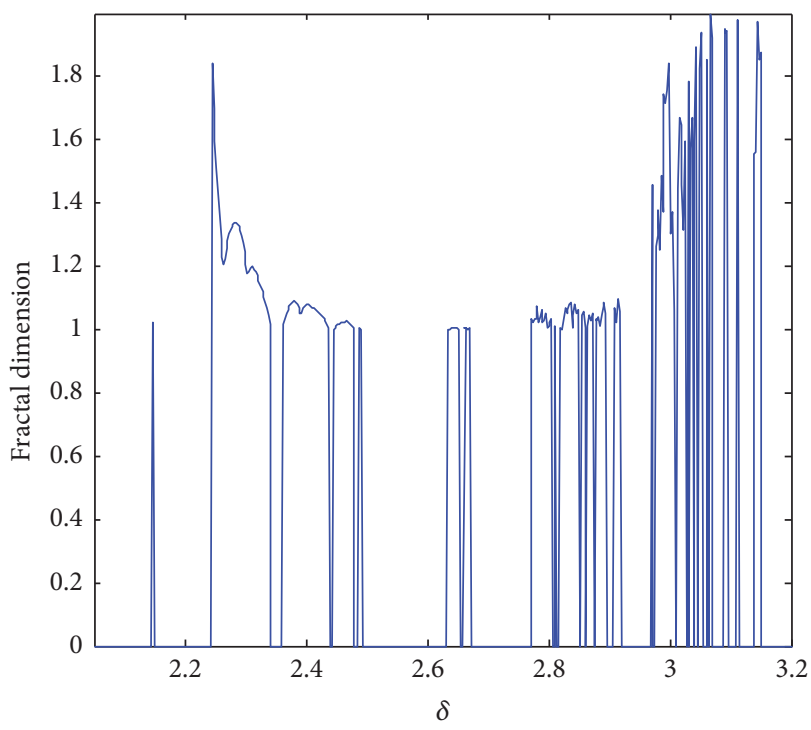

(b)

Figure 7: Fractal dimension of system (4). (a) FD related to Figure 2(a) and (b) FD related to Figure 3(a).

Now, suppose that $\lambda_{1}=1$; then from (57) and (58), we get $\operatorname{tr} J_{c}-\operatorname{det} J_{c}=1$; that is,

$$
\begin{aligned}
l_{2}: & \left(1-a_{22}\right) k_{1}+a_{21} k_{2} \\
& =a_{11}+a_{22}-1-a_{11} a_{22}+a_{12} a_{21} .
\end{aligned}
$$

Next, assume that $\lambda_{1}=-1$; then from (57) and (58), we obtain $\operatorname{tr} J_{c}+\operatorname{det} J_{c}=-1$; that is,

$$
\begin{aligned}
l_{3}: & \left(1+a_{22}\right) k_{1}-a_{21} k_{2} \\
& =a_{11}+a_{22}+1+a_{11} a_{22}-a_{12} a_{21} .
\end{aligned}
$$

Then the lines $l_{1}, l_{2}$, and $l_{3}$ (see Figure $8(\mathrm{a})$ ) in the $\left(k_{1}, k_{2}\right)$ plane determine a triangular region which keeps stable eigenvalues.
In order to observe how feedback method works and controls chaos at unstable state, we have presented some numerical simulations. We set $\delta=3.15$ and the rest as in case(i) as fixed parameters. The initial value is $\left(x_{0}, y_{0}\right)=(0.324,0.21633)$, and the feedback gains are $k_{1}=$ 2.38 and $k_{2}=-1.23$. Figures $8(\mathrm{~b})$ and $8(\mathrm{c})$ illustrate that, at the fixed point $(0.332,0.221333)$, the chaotic trajectory is stabilized.

\section{Discussions}

We investigated the dynamic complexities of discrete predator-prey system with ratio-dependent functional response (4) in the closed first quadrant of $\mathbb{R}_{+}^{2}$. We established 


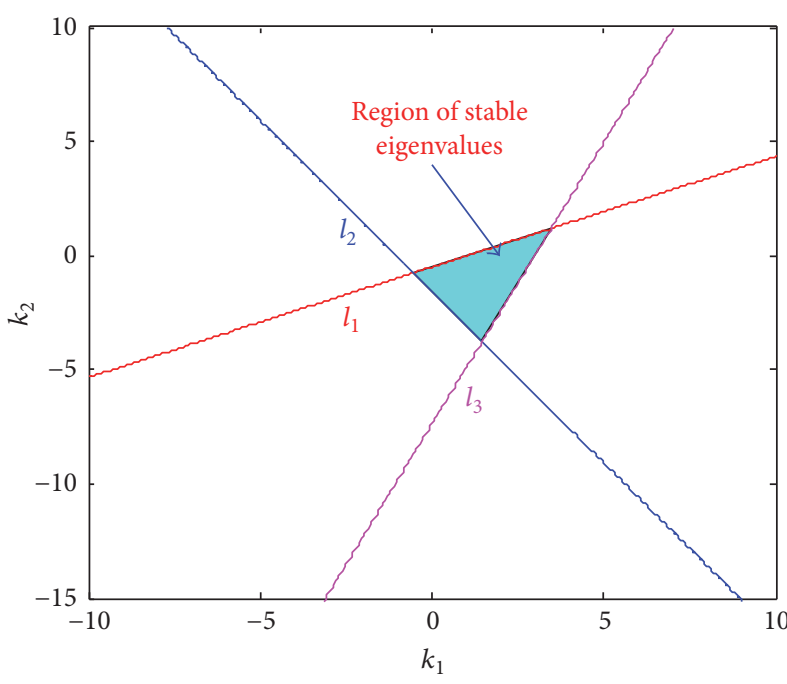

(a)

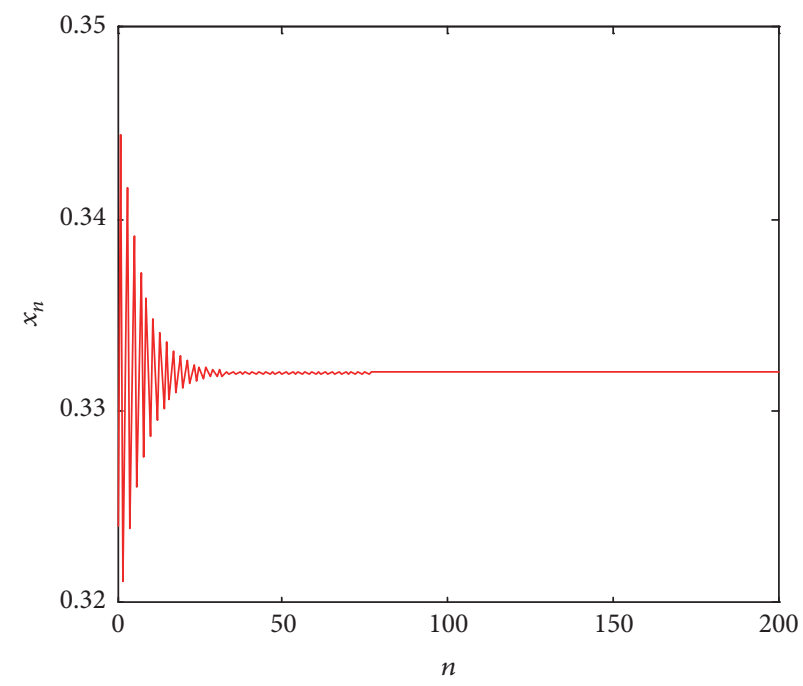

(b)

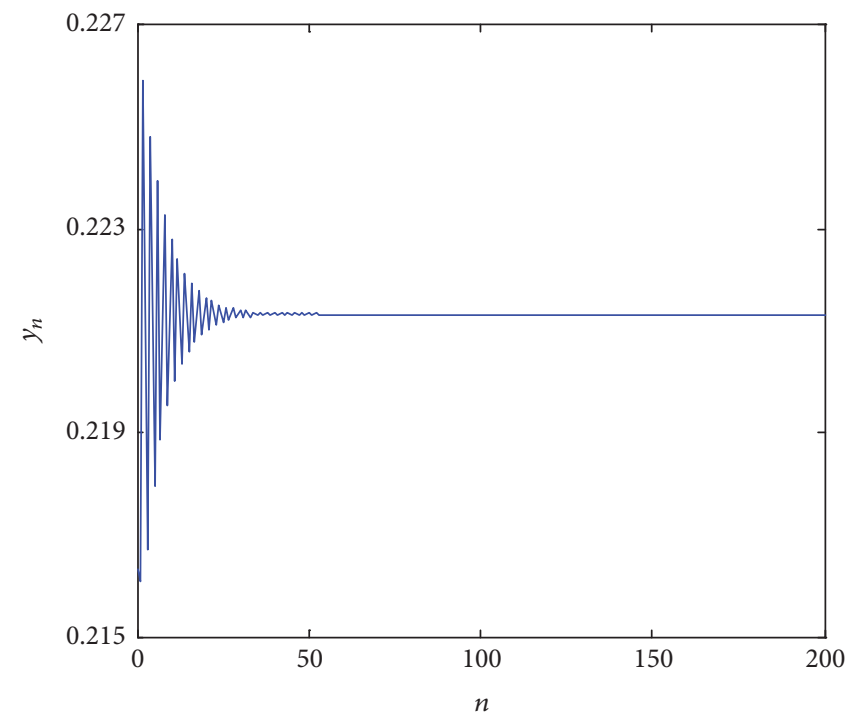

(c)

Figure 8: Control of chaotic trajectories of controlled system (53). (a) Bounded region for stable eigenvalues and (b-c) the time series for states $x$ and $y$.

the existence conditions for a flip bifurcation and a NS bifurcation of system (4) by using center manifold theorem and bifurcation theory. Specifically, we showed that system (4) displays the change of complex dynamical behaviors via flip bifurcation and NS bifurcation including an invariant cycle, orbits of periods 9,19 , and 26 , and attracting chaotic sets when parameter $\delta$ changes its value around sets $\mathrm{FB}_{E_{1}}$ and $\mathrm{NSB}_{E_{2}}$, respectively. This indicates that, at the state of chaos, the system is unstable. In particular, it is observed that if the dynamics of prey is chaotic, then the dynamics of predator will ultimately tend to zero or tend to unstable periodic orbit. This results in the interaction between predator and prey which can approach oscillatory balance behavior. Moreover, the variation of two control parameters which displayed captivating chaotic dynamics exists in the system and one can directly observe the chaotic phenomenon from two-dimensional parameter-spaces. Finally, we applied a strategy of feedback control to stabilize chaos at the state of unstable trajectories resulting in predictable stable orbits.

\section{Conflicts of Interest}

The author declares that there are no conflicts of interest.

\section{References}

[1] A. J. Lotka, Elements of Mathematical Biology, Williams and Wilkins, Baltimore, Md, USA, 1925.

[2] V. Volterra, "Variazioni e fluttuazioni del numero diâindividui in specie animali conviventi," Mem. R. Accad. Naz. Dei Lincei, vol. 2, 1926. 
[3] R. Arditi and L. R. Ginzburg, "Coupling in predator-prey dynamics: ratio-dependence," Journal of Theoretical Biology, vol. 139, no. 3, pp. 311-326, 1989.

[4] A. P. Gutierrez, "The physiological basis of ratio-dependent predatorprey theory: a metabolic pool model of Nicholsonâs blowflies as an example," Ecology, vol. 73, pp. 1552-1563, 1992.

[5] R. Arditi, L. R. Ginzburg, and H. R. Akcakaya, "Variation in plankton densities among lakes: a case for ratio-dependent predation models," The American Naturalist, vol. 138, no. 5, pp. 1287-1296, 1991.

[6] D. Xiao, W. Li, and M. Han, "Dynamics in a ratio-dependent predator-prey model with predator harvesting," Journal of Mathematical Analysis and Applications, vol. 324, no. 1, pp. 1429, 2006.

[7] S.-B. Hsu, T.-W. Hwang, and Y. Kuang, "A ratio-dependent food chain model and its applications to biological control," Mathematical Biosciences, vol. 181, no. 1, pp. 55-83, 2003.

[8] S.-B. Hsu, T.-W. Hwang, and Y. Kuang, "Global analysis of the Michaelis-MENten-type ratio-dependent predator-prey system," Journal of Mathematical Biology, vol. 42, no. 6, pp. 489506, 2001.

[9] D. Xiao and S. Ruan, "Global dynamics of a ratio-dependent predator-prey system," Journal of Mathematical Biology, vol. 43, no. 3, pp. 268-290, 2001.

[10] Z. He and X. Lai, "Bifurcation and chaotic behavior of a discretetime predator-prey system," Nonlinear Analysis. Real World Applications. An International Multidisciplinary Journal, vol. 12, no. 1, pp. 403-417, 2011.

[11] Z. He and B. o. Li, "Complex dynamic behavior of a discretetime predator-prey system of Holling-III type," Advances in Difference Equations, vol. 180, 2014.

[12] Z. Jing and J. Yang, "Bifurcation and chaos in discrete-time predator-prey system," Chaos, Solitons \& Fractals, vol. 27, no. 1, pp. 259-277, 2006.

[13] X. L. Liu and D. M. Xiao, "Complex dynamic behaviors of a discrete-time predator-prey system," Chaos, Solitons \& Fractals, vol. 32, no. 1, pp. 80-94, 2007.

[14] S. M. S. Rana, "Bifurcation and complex dynamics of a discretetime predator-prey system with simplified Monod-Haldane functional response," Advances in Difference Equations, vol. 345, 2015.

[15] S. M. S. Rana, "Bifurcation and complex dynamics of a discretetime predator-prey system involving group defense," Computational Ecology and Software, vol. 5, no. 3, pp. 222-238, 2015.

[16] S. M. S. Rana, "Bifurcation and complex dynamics of a discretetime predator-prey system," Computational Ecology and Software, vol. 5, no. 2, pp. 187-200, 2015.

[17] W. Tan, J. Gao, and W. Fan, "Bifurcation analysis and chaos control in a discrete epidemic system," Discrete Dynamics in Nature and Society, vol. 2015, Article ID 974868, 13 pages, 2015.

[18] C. Wang and X. Li, "Stability and Neimark-Sacker bifurcation of a semi-discrete population model," The Journal of Applied Analysis and Computation, vol. 4, pp. 419-435, 2014.

[19] M. Zhao, Z. Xuan, and C. Li, "Dynamics of a discrete-time predator-prey system," Advances in Difference Equations, vol. 191, 2016.

[20] M. Zhao, C. Li, and J. Wang, "Complex dynamic behaviors of a discrete-time predator-prey system," The Journal of Applied Analysis and Computation, vol. 7, no. 2, pp. 478-500, 2017.

[21] G. Chen, Z. Teng, and Z. Hu, "Analysis of stability for a discrete ratio-dependent predator-prey system," Indian Journal of Pure and Applied Mathematics, vol. 42, no. 1, pp. 1-26, 2011.
[22] L. Cheng and H. Cao, "Bifurcation analysis of a discrete-time ratio-dependent predator-prey model with Allee effect," Communications in Nonlinear Science and Numerical Simulation, vol. 38, pp. 288-302, 2016.

[23] Y. Xia, J. Cao, and M. Lin, "Discrete-time analogues of predatorprey models with monotonic or nonmonotonic functional responses," Nonlinear Analysis: Real World Applications, vol. 8, no. 4, pp. 1079-1095, 2007.

[24] S. N. Elaydi, An Introduction to Difference Equations, SpringerVerlag, New York, NY, USA, 1996.

[25] Y. A. Kuznetsov, Elements of Applied Bifurcation Theory, Springer-Verlag, New York, NY, USA, 2nd edition, 1998.

[26] J. Guckenheimer and P. Holmes, Nonlinear Oscillations, Dynamical Systems, and Bifurcation of Vector Fields, Springer-Verlag, New York, NY, USA, 1983.

[27] C. Robinson, Dynamical Systems: Stability, Symbolic Dynamics and Chaos, Boca Raton, NY, New York, USA, 2nd edition, 1999.

[28] S. Wiggins, Introduction to Applied Nonlinear Dynamical Systems and Chaos, vol. 2, Springer-Verlag, New York, NY, USA, 2003.

[29] J. H. E. Cartwright, "Nonlinear stiffness Lyapunov exponents and attractor dimension," Physics Letters A, vol. 264, pp. 298304, 1999.

[30] J. L. Kaplan and J. A. Yorke, "Preturbulence: a regime observed in a fluid flow model of Lorenz," Communications in Mathematical Physics, vol. 67, no. 2, pp. 93-108, 1979.

[31] S. Lynch, Dynamical Systems with Applications Using Mathematica, Birkhäuser, Boston, Mass, USA, 2007. 


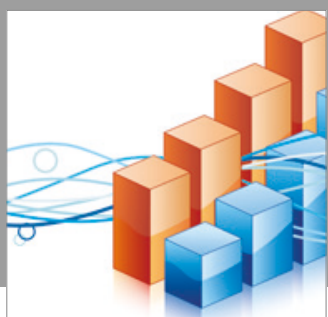

Advances in

Operations Research

vatersals

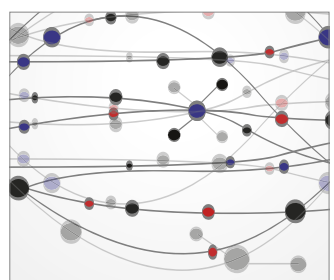

\section{The Scientific} World Journal
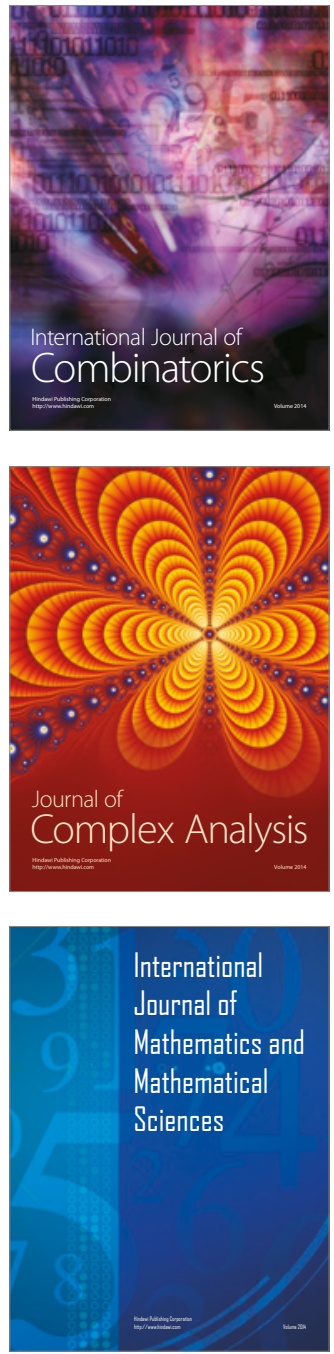
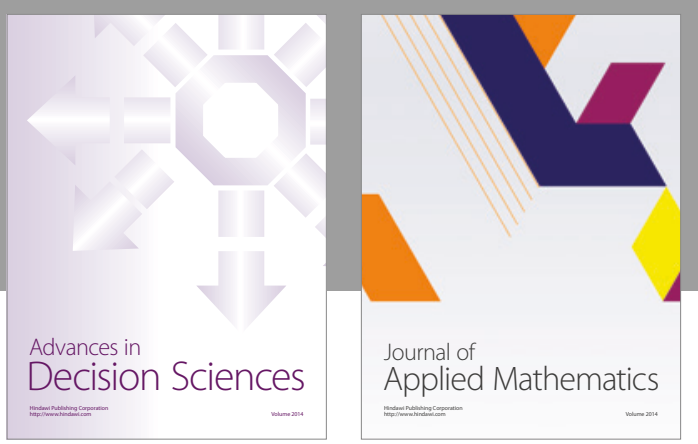

Algebra

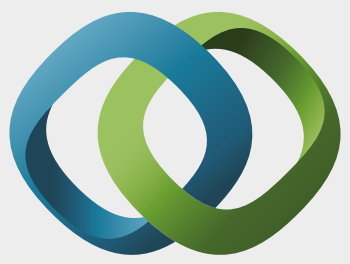

\section{Hindawi}

Submit your manuscripts at

https://www.hindawi.com
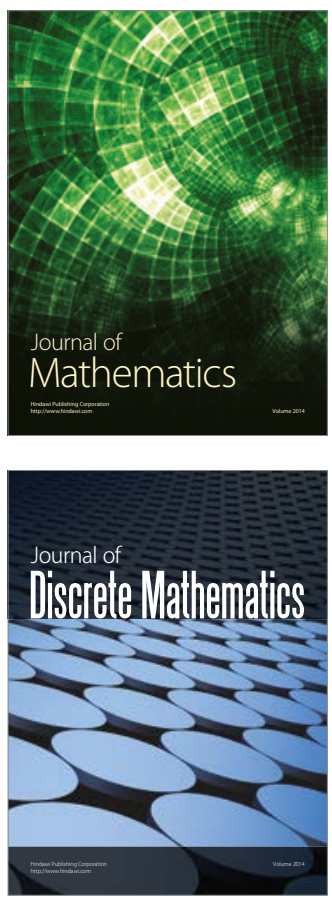

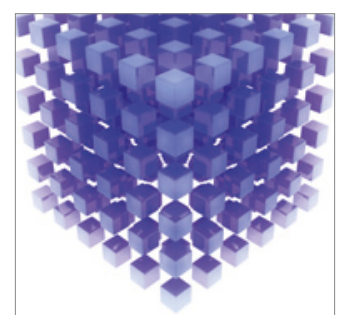

Mathematical Problems in Engineering
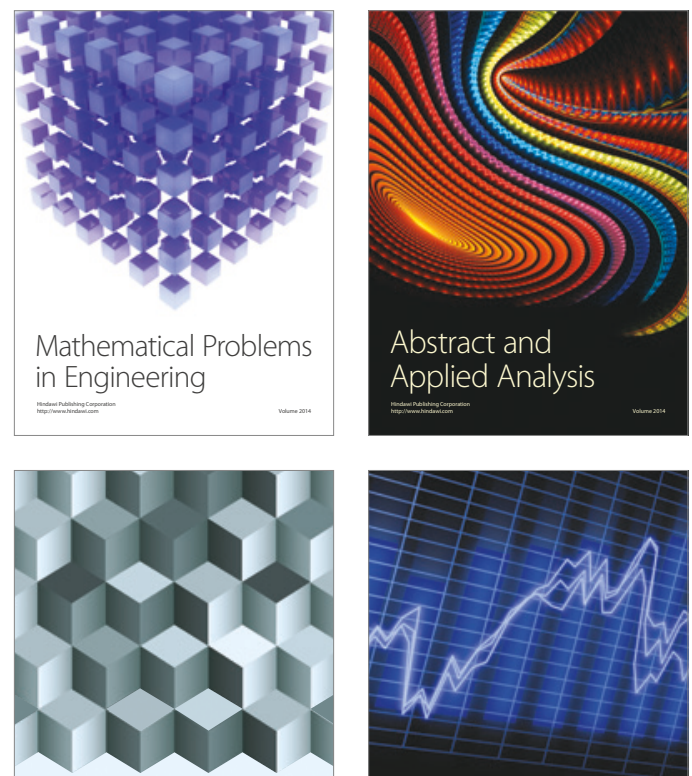

Journal of

Function Spaces

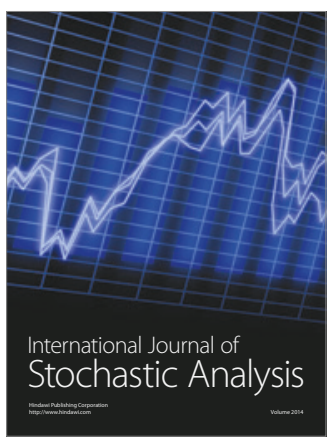

Probability and Statistics
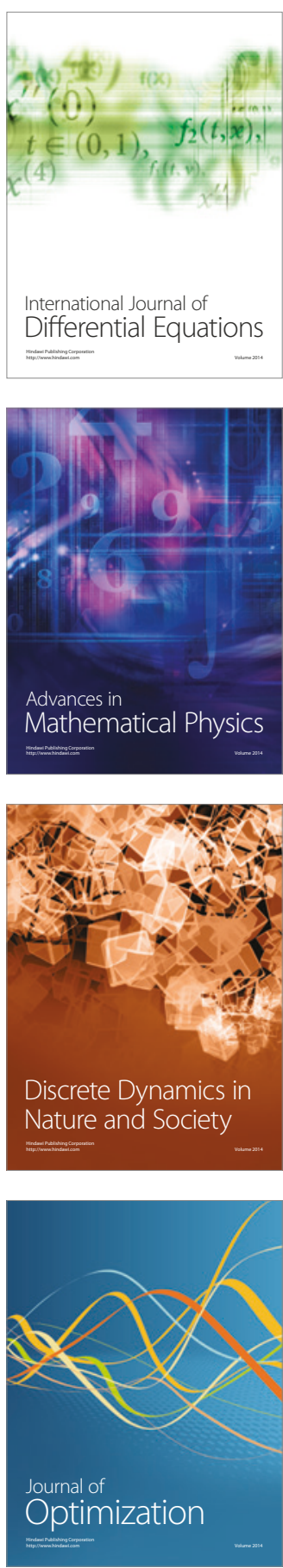